\title{
On the isomorphism problem for generalized Baumslag-Solitar groups
}

\author{
MATT CLAY \\ MAX FORESTER
}

\begin{abstract}
Generalized Baumslag-Solitar groups (GBS groups) are groups that act on trees with infinite cyclic edge and vertex stabilizers. Such an action is described by abeled graph (essentially, the quotient graph of groups). This paper addresses the problem of determining whether two given labeled graphs define isomorphic groups; this is the isomorphism problem for GBS groups. There are two main results and some applications. First, we find necessary and sufficient conditions for a GBS group to be represented by only finitely many reduced labeled graphs. These conditions can be checked effectively from any labeled graph. Then we show that the isomorphism problem is solvable for GBS groups whose labeled graphs have first Betti number at most one.
\end{abstract}

20E08; 20F10, 20F28

\section{Introduction}

A generalized Baumslag-Solitar group (or GBS group) is a group that acts on a tree with infinite cyclic edge and vertex stabilizers. ${ }^{1}$ The tree (together with the group action) is called a GBS tree. A GBS tree can be described by a labeled graph, which is a connected graph whose oriented edges are each labeled by a nonzero integer. This information is enough to specify a graph of groups encoding the GBS tree.

A GBS group $G$ may have many labeled graph descriptions. Even if one restricts to reduced labeled graphs, which are in some sense the simplest ones, there may be infinitely many distinct such graphs defining $G$. It can also happen that there is only one reduced graph, or finitely many. In these latter cases, useful information about $\operatorname{Out}(G)$ can be obtained, as in Gilbert-Howie-Metaftsis-Raptis [8], Pettet [15] and Levitt [13]. Other aspects of GBS groups have been studied by Kropholler, Whyte, Levitt and others. See Kropholler [10; 11], Whyte [16], Clay [2] and Forester [6; 7] for details on various algebraic and geometric properties of GBS groups.

\footnotetext{
${ }^{1}$ In this paper we will only consider finitely generated GBS groups, so finite generation will be added to the definition; see Section 2.2.
} 
The variety of labeled graph descriptions of GBS groups is partly what makes them interesting. For instance, they demonstrate the extent to which JSJ decompositions of groups can fail to be unique. On the other hand, this variety can also be a source of difficulty, such as when studying automorphisms. A given labeled graph need not be invariant, for instance. Even the basic problem of recognizing a given GBS group from one of its labeled graphs is not at all clear.

The isomorphism problem for GBS groups is the problem of determining algorithmically whether two given labeled graphs define isomorphic GBS groups. This problem has only been shown to be solvable in limited special cases. It is trivially solvable for the rigid GBS groups, which are those having a unique reduced labeled graph. These groups were characterized by Levitt [12] (see also Gilbert et al [8], Pettet [15] and Forester [5]).

Levitt showed that the isomorphism problem is solvable in the case of GBS groups $G$ such that $\operatorname{Out}(G)$ does not contain a nonabelian free group [13]. He also solved the isomorphism problem for 2-generator GBS groups [14]. Both of these results rely on having an explicit characterization of the class of groups being considered.

In [7] the second author solved the isomorphism problem for GBS groups whose modular groups contain no integers other than \pm 1 . Equivalently (see Levitt [13]) these are the GBS groups not containing any solvable Baumslag-Solitar group $B S(1, n)$ with $n>1$. It is worth recalling the main steps of the proof. First it was shown that any two such graphs are related by slide moves, without leaving the set of reduced graphs. Then it was shown that such a group is represented by only finitely many reduced labeled graphs. Thus, this set can be searched and enumerated effectively, and membership is decidable.

For the general isomorphism problem, it is useful to understand the space of reduced labeled graphs related to a given one by sequences of slide moves. We want to know whether this space is infinite, and whether it includes all reduced labeled graphs for the given group. To this end, there is a property of edges that plays a key role: edges can be mobile or nonmobile (see Definition 3.12). One of our main technical results is Corollary 3.24, which shows that in any sequence of slide moves, the nonmobile edges may be slid first and one at a time. From this we deduce information on the slide space of a labeled graph, including our first main result:

Theorem 1.1 Let $G$ be a GBS group other than $B S(1, n)$, represented by a reduced labeled graph $\Gamma$. Then $G$ has finite reduced labeled graph space if and only if $\Gamma$ has no mobile edges. 
We also show that mobility of edges can be tested algorithmically (Remark 3.13), so the property of the theorem is decidable. (The case of $B S(1, n)$ is clear as well: the reduced labeled graph is unique if $n \neq-1$, and $B S(1,-1)$ has two reduced labeled graphs.) One consequence of Theorem 1.1 is Theorem 3.29, which solves the isomorphism problem in the case where one labeled graph has no mobile edges.

Next we consider the case of GBS groups whose labeled graphs have first Betti number one. (The Betti number zero case is covered by Forester [7].) The primary goal of the rest of the paper is the following theorem:

Theorem 1.2 There is an algorithm which, given two labeled graphs, one of which has first Betti number at most one, determines whether the two GBS groups are isomorphic.

These are two cases, which behave rather differently: the ascending case and the nonascending case. In the ascending case, there is a structure theorem (Theorem 4.19) which says that the group is uniquely determined by certain invariants, which can be computed by putting the labeled graph into a normal form. These invariants are defined and proved invariant with the aid of Theorem 1.1 of Clay-Forester [4], which shows that any two reduced labeled graphs are related by slide, induction and $\mathscr{A}^{ \pm 1}$-moves between reduced labeled graphs (see Section 2.2 for the definitions of these moves).

The nonascending case is somewhat simpler, since any two reduced labeled graphs representing the same group are related by slide moves. (In particular, one may keep track of individual edges.) However, even though we can define normal forms, they are much less rigid than in the ascending case. For instance, there is no canonical edge with which to compare other edges, unlike ascending normal forms.

We show that given $G$, there are only finitely many reduced labeled graphs in normal form, and these can be enumerated effectively. The solution to the isomorphism problem is then similar to the case proved in [7].

Acknowledgements The second author is partially supported by NSF grant DMS0605137.

\section{Preliminaries}

\subsection{Deformation spaces}

A graph $\Gamma$ is given by $\left(V(\Gamma), E(\Gamma), o, t,{ }^{-}\right)$where $V(\Gamma)$ are the vertices, $E(\Gamma)$ are the oriented edges, $o, t: E(\Gamma) \rightarrow V(\Gamma)$ are the originating and terminal vertex maps 
and $^{-}: E(\Gamma) \rightarrow E(\Gamma)$ is a fixed point free involution, which reverses the orientations of edges. An edge path $\gamma=\left(e_{0}, \ldots, e_{k}\right)$ is a sequence of edges such that $t\left(e_{i}\right)=o\left(e_{i+1}\right)$ for $i=0, \ldots, k-1$. A loop is an edge $e \in E(\Gamma)$ such that $o(e)=t(e)$. A geometric $e d g e$ is a pair of the form $\{e, \bar{e}\}$.

Let $G$ be a group. A $G$-tree is a simplicial tree $T$ together with an action of $G$ by simplicial automorphisms, without inversions (that is, $g e \neq \bar{e}$ for all $g \in G, e \in E(T)$ ). Two $G$-trees are considered equivalent if there is a $G$-equivariant isomorphism between them. The quotient graph $T / G$ has the structure of a graph of groups with a marking (an identification of $G$ with the fundamental group of the graph of groups).

Given a $G$-tree $T$, a subgroup $H \subseteq G$ is elliptic if it fixes a point of $T$. There are two moves one can perform on a $G$-tree without changing the elliptic subgroups, called collapse and expansion moves; they correspond to the natural isomorphism $A *_{B} B \cong A$. The exact definition is as follows.

Definition 2.1 An edge $e$ in a $G$-tree $T$ is collapsible if $G_{e}=G_{o(e)}$ and its endpoints are not in the same orbit. If one collapses $\{e, \bar{e}\}$ and all of its translates to vertices, the resulting $G$-tree is said to be obtained from $T$ by a collapse move. The reverse of this move is called an expansion move.

A $G$-tree is reduced if it does not admit a collapse move. An elementary deformation is a finite sequence of collapse and expansion moves. Given a $G$-tree $T$, the deformation space $\mathscr{D}$ of $T$ is the set of all $G$-trees related to $T$ by an elementary deformation. If $T$ is cocompact then $\mathscr{D}$ is equivalently the set of all $G$-trees having the same elliptic subgroups as $T$, by Forester [5].

\subsection{Generalized Baumslag-Solitar groups}

A group $G$ that acts on a tree with infinite cyclic stabilizers is called a generalized Baumslag-Solitar group (or GBS group). In this paper, for simplicity, we also require $G$ to be finitely generated (this convention is not followed in $[5 ; 6 ; 7]$ ). The tree is called a $G B S$ tree. In the quotient graph of groups, every vertex and edge group is isomorphic to $\mathbb{Z}$, and each inclusion map $G_{e} \hookrightarrow G_{o(e)}$ is given by multiplication by a nonzero integer. This data can be effectively represented in a labeled graph. Specifically, a labeled graph is a pair $(\Gamma, \lambda)$ where $\Gamma$ is a finite connected graph and $\lambda: E(\Gamma) \rightarrow \mathbb{Z}-\{0\}$ is a function, called the labeling. Given a choice of generators of $G_{e}$ and $G_{o(e)}$, the map $G_{e} \hookrightarrow G_{o(e)}$ is multiplication by $\lambda(e)$. Replacing a generator of an edge group $G_{e}$ by its inverse interchanges the signs of $\lambda(e)$ and $\lambda(\bar{e})$; replacing a generator of a vertex group $G_{v}$ by its inverse interchanges the signs of $\lambda(e)$ for all edges $e$ originating at $v$. 
These operations are called admissible sign changes. This is the only ambiguity in the labels of a labeled graph. We will sometimes refer to $(\Gamma, \lambda)$ simply as $\Gamma$.

A $G$-tree is elementary if there is a $G$-invariant point or line, and is nonelementary otherwise. By Forester [6, Lemma 2.6], a GBS tree is elementary if and only if the group is isomorphic to $\mathbb{Z}, \mathbb{Z} \times \mathbb{Z}$, or the Klein bottle group. Thus a GBS group not isomorphic to one of these three groups is called a nonelementary GBS group.

In a nonelementary GBS group, the elliptic subgroups arising from any GBS tree are characterized algebraically [6, Lemma 2.5]. Therefore, any two such $G$-trees lie in the same deformation space. In particular, any two labeled graphs representing the same nonelementary group are related by an elementary deformation. Whenever we speak of a deformation space for a nonelementary GBS group, we will always be referring to this canonical deformation space. For a description of this canonical deformation space associated to the classical Baumslag-Solitar groups $B S(p, q)$, see Clay [2]. Unless otherwise stated, all GBS groups considered here will be assumed to be nonelementary.

In a labeled graph, a loop $e$ with label \pm 1 is called an ascending loop. It is a strict ascending loop if $\lambda(\bar{e}) \neq \pm 1$. A loop $e$ is a virtual ascending loop if $\lambda(e)$ divides $\lambda(\bar{e})$, and is a strict virtual ascending loop if, in addition, $\lambda(\bar{e}) \neq \pm \lambda(e)$. A GBS deformation space is ascending if it contains a GBS tree whose labeled graph has a strict ascending loop. We also say that $G$ is ascending. Otherwise the deformation space (or the group) is called nonascending.

Now we define various moves between GBS trees, all of which are elementary deformations. The moves in Definition 2.2 are discussed more fully (in the general setting of $G$-trees) in [7]. In particular, slides and inductions can be factored as an expansion followed by a collapse. A general discussion of $\mathscr{A}^{ \pm 1}$-moves can be found in Clay-Forester [4].

In the diagrams below, each label $\lambda(e)$ is pictured next to the endpoint $o(e)$. We begin with the elementary moves, which look as follows (modulo admissible sign changes):

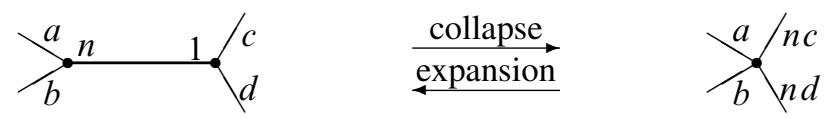

Thus a GBS tree is reduced if and only if its labeled graph does not contain an edge with distinct endpoints and label \pm 1 . 
Definition 2.2 A slide move between GBS trees takes one of the following two forms:

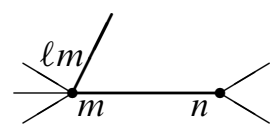

slide

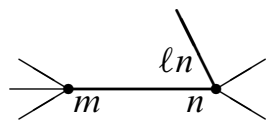

or

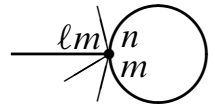

slide

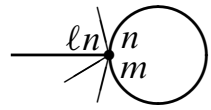

An induction move between GBS trees is as follows:

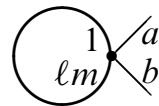

$\stackrel{\text { induction }}{\longrightarrow}$

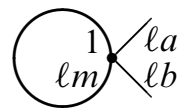

Both directions of the move are considered induction moves. This move decomposes into an elementary deformation as follows:

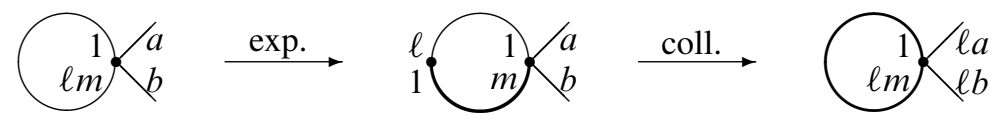

Definition 2.3 Next we discuss $\mathscr{A}^{ \pm 1}$-moves, defined in [4]. An $\mathscr{A}^{-1}$-move is an induction followed by a collapse, with the following description. It is required that $k, \ell \neq \pm 1$, and that the left hand vertex has no other edges incident to it.
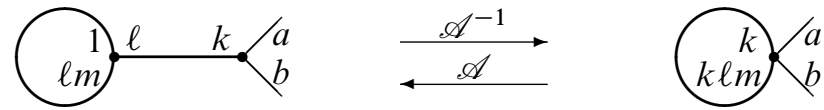

The induction move changes the label $\ell$ to 1 , after which the edge is collapsed.

Note that before the move, the loop is a strict ascending loop, and after, the loop is not ascending. Thus an $\mathscr{A}^{-1}$-move removes an ascending loop, and its reverse, called an $\mathscr{A}$-move, adds one.

Remark 2.4 $\mathscr{A}^{ \pm 1}$-moves preserve the property of being reduced. The same is not always true of slide or induction moves, unless one is in a nonascending deformation space. Also, an induction or $\mathscr{A}^{ \pm 1}$-move can only occur in an ascending deformation space. 
We will make extensive use of the following result, which is the main theorem of [4], and its corollary.

Theorem 2.5 In a deformation space of cocompact $G$-trees, any two reduced trees are related by a finite sequence of slides, inductions, and $\mathscr{A}^{ \pm 1}$-moves, with all intermediate trees reduced.

Corollary 2.6 In a nonascending deformation space of cocompact $G$-trees, any two reduced trees are related by a finite sequence of slide moves, with all intermediate trees reduced. Moreover, if $e$ is an edge of $T$ and a deformation from $T$ to $T^{\prime}$ never collapses $e$, then there is a sequence of slide moves from $T$ to $T^{\prime}$ in which no edge slides over $e$.

The first statement of the corollary follows immediately from the theorem, and has previously appeared as Forester [7, Theorem 7.4] and Guirardel-Levitt [9, Theorem 7.2]. The second statement is proved in [4].

\subsection{The modular homomorphism}

Let $G$ be a GBS group with labeled graph $(\Gamma, \lambda)$. There are two versions of the modular homomorphism $G \rightarrow \mathbb{Q}^{\times}$, each with several descriptions; see Bass-Kulkarni [1], Forester [7] and Kropholler [11]. In this paper, it turns out to be more convenient to use the reciprocal of the usual definition, so we will include this modification here. This makes it easier to keep track of slide moves; see for example Definition 3.2 and Remark 3.3. We will mostly work with the signed modular homomorphism $q: G \rightarrow \mathbb{Q}^{\times}$, defined as the composition $G \rightarrow H_{1}(\Gamma) \rightarrow \mathbb{Q}^{\times}$where the second map is given by

$$
\left(e_{1}, \ldots, e_{k}\right) \mapsto \prod_{i=1}^{k} \frac{\lambda\left(\bar{e}_{i}\right)}{\lambda\left(e_{i}\right)} .
$$

(The first map is given by first killing the normal closure of the elliptic elements to obtain $\pi_{1}(\Gamma)$, and then abelianizing.) Equivalently, fix a nontrivial elliptic element $a \in G$. Then every $g \in G$ satisfies a relation $g a^{r} g^{-1}=a^{s}$ in $G$ for some nonzero integers $r$ and $s$, and the assignment $q(g)=s / r$ is a well defined homomorphism, which agrees with the definition just given; see Kropholler [11] or Levitt [13].

The unsigned modular homomorphism is simply $|q|$, defined on $H_{1}(\Gamma)$ by

$$
\left(e_{1}, \ldots, e_{k}\right) \mapsto \prod_{i=1}^{k} \frac{\left|\lambda\left(\bar{e}_{i}\right)\right|}{\left|\lambda\left(e_{i}\right)\right|}
$$


An equivalent definition is to choose any subgroup $V$ of $G$ commensurable with a vertex group, and assign to each $g \in G$ the positive rational number

$$
\left[V^{g}: V \cap V^{g}\right] /\left[V: V \cap V^{g}\right] .
$$

See Forester [7] for a proof that this function agrees with $|q(g)|$. We say that $(\Gamma, \lambda)$ is unimodular if $|q|$ is trivial.

Finally, there is also an orientation homomorphism $G \rightarrow\{ \pm 1\}$ defined by $g \mapsto$ $q(g) /|q(g)|$. This homomorphism is also defined on $H_{1}(\Gamma)$. The next result shows that the GBS group associated to a labeled graph is determined by the orientation homomorphism and the absolute value of the labeling. Hence it often suffices to consider positive labeled graphs, ie labeled graphs $(\Gamma, \lambda)$ such that $\lambda(e)>0$ for all $e \in E(\Gamma)$.

Lemma 2.7 Let $\lambda$ and $\lambda^{\prime}$ be labelings on a graph $\Gamma$ such that $|\lambda|=\left|\lambda^{\prime}\right|$. If their orientation homomorphisms agree then $(\Gamma, \lambda)$ and $\left(\Gamma, \lambda^{\prime}\right)$ differ by admissible sign changes. In particular, the corresponding GBS groups are isomorphic.

Proof Let $\Omega: H_{1}(\Gamma) \rightarrow\{ \pm 1\}$ be the orientation homomorphism of $(\Gamma, \lambda)$ and $\left(\Gamma, \lambda^{\prime}\right)$. Fix a maximal tree $T \subseteq \Gamma$. Then every edge $e$ of $\Gamma-T$ determines a generator $[e] \in H_{1}(\Gamma)$.

By admissible sign changes, we can arrange that $\lambda$ and $\lambda^{\prime}$ agree, and are positive, on the edges of $T$. Then for any edge $e$ in $\Gamma-T$ we have that $\Omega([e])=1$ if and only if $\lambda(e)$ and $\lambda(\bar{e})$ have the same sign, if and only if $\lambda^{\prime}(e)$ and $\lambda^{\prime}(\bar{e})$ have the same sign. Thus $\lambda$ and $\lambda^{\prime}$ can be made to agree on $e$ and $\bar{e}$ by an admissible sign change affecting $e, \bar{e}$ only. In this way, $\lambda$ and $\lambda^{\prime}$ can be made to agree on all of $\Gamma$.

\section{Labeled graph spaces}

From now on we consider only GBS groups and their canonical deformation spaces. Hence we will always refer to $G$ instead of this deformation space.

Definition 3.1 For a GBS group $G$, we denote by $\operatorname{RLG}(G)$ the set of reduced labeled graphs representing $G$. Let $\mathrm{RLG}^{+}(G)$ be the set of positive reduced labeled graphs representing $G$. Note that this latter set is nonempty only if the orientation homomorphism is trivial.

Our goal in this section is to establish a criterion, which can be checked in terms of any labeled graph in $\operatorname{RLG}(G)$, that characterizes when $\operatorname{RLG}(G)$ is finite. Notice 
that if $G$ is ascending and $G \neq B S(1, n)$, then $|\operatorname{RLG}(G)|=\infty$. If $G=B S(1, n)$ or $G=\mathbb{Z}$, then $\operatorname{RLG}(G)$ consists of a single point (unless $G=B S(1,-1)$, in which case $|\operatorname{RLG}(G)|=2$ ). Therefore, we are mainly concerned with determining when a nonascending GBS group satisfies $|\operatorname{RLG}(G)|=\infty$. However, we will need to prove a more general statement, as we do not have an algorithm to determine whether a given GBS group is ascending.

\subsection{Monotone cycles and mobile edges}

If $\left(e_{0}, \ldots, e_{n}\right)$ is an edge path in $\Gamma$, we define $q\left(e_{0}, \ldots, e_{n}\right)$ by formula (1). This is also denoted $q_{\Gamma}\left(e_{0}, \ldots, e_{n}\right)$.

Definition 3.2 Let $\Gamma$ be a labeled graph for $G$ and $e \in E(\Gamma)$. An edge path $\left(e_{0}, \ldots, e_{n}\right)$ is an $e-e d g e$ path if

(a) $e_{i} \neq e, \bar{e}$ for $i=0, \ldots, n$;

(b) $o(e)=o\left(e_{0}\right)$; and

(c) $\lambda\left(e_{i}\right)$ divides $\lambda(e) q\left(e_{0}, \ldots, e_{i-1}\right)$ for $i=0, \ldots, n$.

An $e$-edge path is an $e$-integer cycle if, in addition we have

(d) $o\left(e_{0}\right)=t\left(e_{n}\right)$; and

(e) $q\left(e_{0}, \ldots, e_{n}\right) \in \mathbb{Z}$.

If $\left|q\left(e_{0}, \ldots, e_{n}\right)\right| \neq 1$ we say that the $e$-edge path or the $e$-integer cycle is strict.

Remark 3.3 The first three conditions are necessary and sufficient to be able to slide $e$ along $\left(e_{0}, \ldots, e_{n}\right)$. The resulting label on the edge $e$ is $\lambda(e) q\left(e_{0}, \ldots, e_{n}\right)$. Hence $e$ may slide repeatedly along an $e$-integer cycle. Also notice that any path obtained by tightening an $e$-edge path (respectively, $e$-integer cycle) is an $e$-edge path (respectively, $e$-integer cycle).

Definition 3.4 An edge path $\left(e_{0}, \ldots, e_{n}, e\right)$ is a monotone cycle if $\left(e_{0}, \ldots, e_{n}\right)$ is an $\bar{e}$-edge path and $q\left(e_{0}, \ldots, e_{n}, e\right) \in \mathbb{Z}$. An edge $e$ is a monotone cycle if $e$ is a loop and $q(e) \in \mathbb{Z}$. A monotone cycle is strict if the modulus is not equal to \pm 1 .

Remark 3.5 Suppose $\left(e_{0}, \ldots, e_{n}, e\right)$ is a monotone cycle. Since $\left(e_{0}, \ldots, e_{n}\right)$ is an $\bar{e}$-edge path, $o\left(e_{0}\right)=o(\bar{e})=t(e)$. Hence a monotone cycle is a cycle. Further, since $\bar{e} \notin\left(e_{0}, \ldots, e_{n}, e\right)$, it is a nontrivial cycle. Notice that in the definition of monotone cycle, the final edge is distinguished. In particular, a cyclic reordering of the edges in a monotone cycle may not be a monotone cycle. 
Lemma 3.6 If $\Gamma$ has a strict monotone cycle, then $G$ is ascending. Further, if $\Gamma$ has a strict monotone cycle, then $\Gamma$ has an immersed strict monotone cycle.

Proof If $\Gamma$ contains a strict monotone cycle which is a single edge $e$, then either $e$ is a strict ascending or strict virtual ascending loop. Therefore, after an $\mathscr{A}$-move in the second case, we see that $G$ is ascending. Otherwise, suppose $\left(e_{0}, \ldots, e_{n}, e\right)$ is a strict monotone cycle in $\Gamma$. Then we can slide $\bar{e}$ along $\left(e_{0}, \ldots, e_{n}\right)$, turning $e$ into a loop. After the slide move, the modulus of the loop is a nontrivial integer, hence $e$ is either a strict ascending or strict virtual ascending loop. As before, this shows that $G$ is ascending.

For the second statement in the lemma, we must show that after tightening, a monotone cycle is still a monotone cycle. This is clear since if $\left(e_{0}, \ldots, e_{n}, e\right)$ is a monotone cycle, then $e_{i} \neq e, \bar{e}$. Therefore, after tightening, the edge $e$ remains in the cycle and the only tightening occurs in the edge path $\left(e_{0}, \ldots, e_{n}\right)$, which remains an $\bar{e}$-edge path after tightening.

Example 3.7 The converse to the first statement of Lemma 3.6 does not hold in general, though we shall prove it in a special case in Proposition 4.2. A counterexample is given by the labeled graphs in Figure 1. The labeled graphs in this figure represent the same GBS group; the labeled graph on the right is obtained by sliding $e_{3}$ over $\bar{e}$. The labeled graph on the left contains a strict monotone cycle, namely the cycle $\left(e_{1}, e_{2}, e_{3}, e\right)$. After sliding $\bar{e}$ over $\left(e_{1}, e_{2}, e_{3}\right), e$ is a virtual ascending loop with labels $\lambda(e)=6, \lambda(\bar{e})=132$.
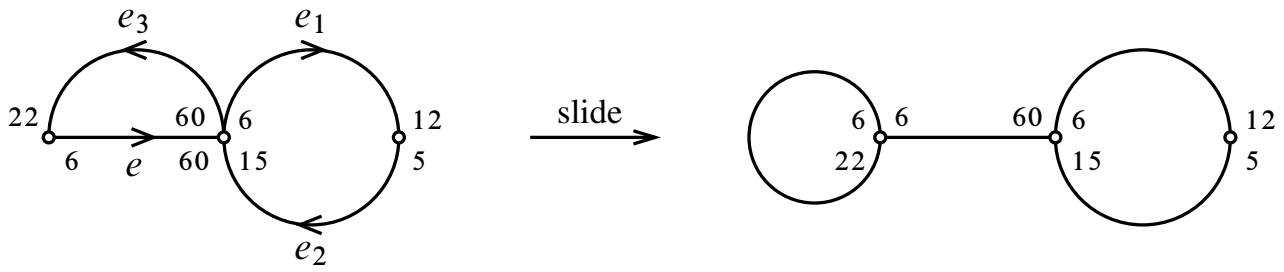

Figure 1: Sliding $e_{3}$ over $\bar{e}$ results in a graph with no strict monotone cycles

We claim that the labeled graph on the right does not have any strict monotone cycles. First, notice that none of the edges $e_{1}, \bar{e}_{1}, e_{2}, \bar{e}_{2}$ can slide. Also, since $e$ is separating (a fact that remains true after sliding $e$ or $\bar{e}$ ), no strict monotone cycle can end with $e$ or $\bar{e}$. Finally, notice that $\bar{e}_{3}$ cannot slide. Hence, if there is a strict monotone cycle, it must be of the form $\left(\alpha, \bar{e}_{3}\right)$, where $\alpha$ is an $e_{3}$-edge path. In particular, $\lambda\left(e_{3}\right) q(\alpha)$ must be divisible by $\lambda\left(\bar{e}_{3}\right)=22$. However, the only place the prime number 11 appears in the labeled graph is in the label $\lambda\left(\bar{e}_{3}\right)$, and since $\bar{e}_{3} \notin \alpha, \lambda\left(e_{3}\right) q(\alpha)$ is not divisible 
by 11 for any $e_{3}$-edge path $\alpha$. Therefore, the labeled graph on the right cannot contain a strict monotone cycle.

Remark 3.8 In general, finding a monotone cycle requires a solution to the conjugacy problem for GBS groups (see Lemma 3.14). This problem is not yet known to be solvable.

Definition 3.9 Given $\Gamma \in \operatorname{RLG}(G)$ and $e \in E(\Gamma)$, we denote by $\mathrm{S}(\Gamma, e) \subseteq \mathrm{RLG}(G)$ the set of reduced labeled graphs obtained from $\Gamma$ by a sequence of slides of $e$ and $\bar{e}$. $\mathrm{S}(\Gamma, e)$ is then called the slide space of $e$ (based at $\Gamma$ ).

Proposition 3.10 Let $\Gamma \in \operatorname{RLG}(G)$ and $e \in E(\Gamma)$. Then $|\mathrm{S}(\Gamma, e)|=\infty$ if and only if $\Gamma$ contains a strict $e$-integer cycle or a strict $\bar{e}-$ integer cycle.

Proof By Remark 3.3, it is clear that if $\Gamma$ contains a strict $e$-integer cycle or a strict $\bar{e}$-integer cycle, then $|\mathrm{S}(\Gamma, e)|=\infty$.

For the converse let $\Gamma_{i}$ be an infinite sequence of labeled graphs in $\mathrm{S}(\Gamma, e)$. As the number of edges in the graphs $\Gamma_{i}$ is constant, there is a subsequence such that $\Gamma_{i}=\Gamma^{\prime}$ (as unlabeled graphs) for some fixed graph $\Gamma^{\prime}$. Thus either $\left|\lambda_{i}(e)\right|$ or $\left|\lambda_{i}(\bar{e})\right|$ is an unbounded sequence of natural numbers. By interchanging $e$ for $\bar{e}$ and passing to a subsequence if necessary, we can assume that $\left|\lambda_{i}(e)\right|$ is a strictly increasing sequence of natural numbers. Since slides of $e$ commute with slides of $\bar{e}$, we can assume that the labeled graphs $\Gamma_{i}$ are obtained from each other without sliding $\bar{e}$. There is a finite number of primes appearing in the sequence $\left\{\left|\lambda_{i}(e)\right|\right\}$. Indeed, this list is contained in the set of primes that appear on any labeled graph for $G$. Therefore, by the following lemma, there are $n, n^{\prime}$ such that $\lambda_{n}(e)$ divides $\lambda_{n^{\prime}}(e)$. Let $\gamma$ be the $e$-edge path that $e$ slid along transforming $\Gamma$ into $\Gamma_{n}$ and $\gamma^{\prime}$ the strict $e$-integer cycle that $e$ slid along transforming $\Gamma_{n}$ into $\Gamma_{n^{\prime}}$. Then clearly $\gamma \gamma^{\prime} \bar{\gamma}$ is a strict $e$-integer cycle in $\Gamma$.

Lemma 3.11 Let $\left\{m_{i}\right\}$ be a strictly increasing sequence of natural numbers such that only finitely many primes appear in the sequence. Then there are distinct indices $n, n^{\prime}$ such that $m_{n}$ divides $m_{n^{\prime}}$.

Proof We will prove this by induction on the number of primes appearing in the sequence $\left\{m_{i}\right\}$. If there is only one prime appearing, then the lemma is obvious.

Suppose that $N$ primes appear in the sequence $\left\{m_{i}\right\}$. To any element $m$ in the sequence we associate a point in $\mathbb{Z}_{\geqslant 0}^{N}$ (ie, the first orthant of $\mathbb{Z}^{N}$ ) by:

$$
\prod_{j=1}^{N} p_{j}^{k_{j}} \mapsto\left(k_{1}, \ldots, k_{N}\right)
$$


where $\prod_{j=1}^{N} p_{j}^{k_{j}}$ is the prime decomposition of $m$. For any element $m_{i}$ in the sequence, we denote the $j$-th coordinate in this assignment by $\left(m_{i}\right)_{j}$. If there is some element $m_{i}$ such that $\left(m_{1}\right)_{j} \leqslant\left(m_{i}\right)_{j}$ for all $j$, then $m_{1}$ divides $m_{i}$ and the conclusion of the lemma holds.

Otherwise, by passing to a subsequence, we can assume that $\left(m_{i}\right)_{j}<\left(m_{1}\right)_{j}$ for some fixed $j$ and all $i$. By further passing to a subsequence we can assume that $\left(m_{i}\right)_{j}=M$ for all $i$. Then $\left\{m_{i} / p_{j}^{M}\right\}$ is a strictly increasing sequence of natural numbers in which only $N-1$ primes appear. Now apply induction to complete the proof.

Definition 3.12 Let $\Gamma \in \operatorname{RLG}(G)$. An edge $e \in E(\Gamma)$ is mobile if either

(a) there is a strict monotone cycle of the form $\left(e_{0}, \ldots, e_{n}, e\right)$ or $\left(e_{0}, \ldots, e_{n}, \bar{e}\right)$; or

(b) $|\mathrm{S}(\Gamma, e)|=\infty$ (equivalently, by Proposition 3.10, $\Gamma$ contains a strict $e$-integer cycle or a strict $\bar{e}$-integer cycle).

An edge that is not mobile is called nonmobile. Note that mobility is a property of geometric edges: $e$ is mobile if and only $\bar{e}$ is.

Remark 3.13 By Proposition 3.10 there is an algorithm to determine whether a given edge $e \in E(\Gamma)$ is mobile or not. Indeed, given an edge we can start making an exhaustive search of $\mathrm{S}(\Gamma, e)$. Either this space is finite or we find an strict $e$-integer cycle or strict $\bar{e}$-integer cycle. In the latter case, $e$ is mobile. If the slide space is finite, we can search these graphs to see if $e$ is a strict ascending or strict virtual ascending loop in any of the graphs. An affirmative answer implies that $e$ is mobile, a negative answer implies that $e$ is nonmobile.

Let $T$ denote the Bass-Serre tree covering $\Gamma$.

Lemma 3.14 An edge $e \in E(\Gamma)$ is mobile if and only if $G_{\widetilde{e}}^{t} \subsetneq G_{\widetilde{e}}$ for some $t \in G$ and some lift $\tilde{e} \in E(T)$ of $e$.

Proof It is clear that if $e$ or $\bar{e}$ is the last edge of a strict monotone cycle, or there is strict $e$-integer cycle or strict $\bar{e}$-integer cycle then there is a lift $\widetilde{e}$ and a $t \in G$ satisfying the conclusion of the lemma.

For the converse, given $t$ and $\widetilde{e}$ with $G_{\widetilde{e}}^{t} \subsetneq G_{\tilde{e}}$, we can replace $t$ if needed to arrange that there are no $G$-translates of $\widetilde{e}$ along the edge path connecting $\widetilde{e}$ to $t \tilde{e}$. Also we can assume without loss of generality that $o(\widetilde{e})$ separates $\widetilde{e}$ from $t \widetilde{e}$. Let $\tilde{\alpha}$ be the path in $T$ from $\tilde{e}$ to $t \tilde{e}$, and let $\alpha$ be its image in $\Gamma$.

If $\tilde{e}$ and $t \tilde{e}$ are coherently oriented, then $(\alpha, \bar{e})$ is a strict monotone cycle. Otherwise, $\alpha$ is a strict $e$-integer cycle. 
Corollary 3.15 If $\Gamma, \Gamma^{\prime} \in \operatorname{RLG}(G)$ are related by slide moves and $e \in E(\Gamma)$ is mobile, then $e$ is mobile in $\Gamma^{\prime}$.

Proof This follows from Lemma 3.14 since edge stabilizers are unchanged by slide moves.

Example 3.7 shows that both parts of the definition for mobility are needed. The edge $e$ is part of a strict monotone cycle $\left(e_{1}, e_{2}, e_{3}, e\right)$ in the labeled graph on the left and hence is mobile. In the labeled graph on the right, as noted in the example, there are no strict monotone cycles, but there is a strict $\bar{e}$-integer cycle, so $e$ is mobile.

Remark 3.16 The set of nonmobile edges is preserved by slides, inductions, and $\mathscr{A}^{ \pm 1}$-moves. To make sense of the third case, observe that even though an $\mathscr{A}^{ \pm 1}-$ move changes the set of edges, the edges directly involved in the move are all mobile, so each nonmobile edge is present before and after the move, and its status does not change (by Lemma 3.14). In the case of an induction move, the loop is mobile before and after, and mobility of other edges is not affected, again by Lemma 3.14. Therefore, for any labeled graph space, we can compare nonmobile edges between any two labeled graphs.

Lemma 3.17 In a labeled graph, a nonmobile edge cannot slide over a mobile edge.

Proof Suppose an edge $f$ slides over a mobile edge $e$. Then there are lifts $\tilde{f}$ and $\tilde{e}$ in the covering tree $T$ such that $\tilde{f}$ slides over $\widetilde{e}$, and so $G_{\tilde{f}} \subseteq G_{\tilde{e}}$. Let $n=\left[G_{\tilde{e}}: G_{\tilde{f}}\right]$. By Lemma 3.14 there is a $t \in G$ such that $G_{\widetilde{e}}^{t} \subsetneq G_{\widetilde{e}}$. Let $m=\left[G_{\widetilde{e}}: G_{\widetilde{e}}^{t}\right]$. Then

$$
G_{\tilde{f}}^{t} \subseteq G_{\widetilde{e}}^{t} \subsetneq G_{\widetilde{e}}
$$

and $G_{\widetilde{f}}^{t}$ is the unique subgroup of $G_{\widetilde{e}}$ of index $m n$. This implies that $G_{\widetilde{f}}^{t}$ is the subgroup of $G_{\tilde{f}}$ of index $m$, which is greater than 1 , and so $f$ is mobile.

\subsection{Slide relations}

In this subsection we will work out some methods to rearrange sequences of slide moves. In particular, we will show that any sequence of slides can be rewritten so that nonmobile edges slide before mobile edges, and individual nonmobile edges can be slid one at a time. To simplify the discussion, we will only consider positive labeled graphs. All slides in this section are between reduced trees (that is, the slides take place "in $\operatorname{RLG}^{+}(G)$ "). 
Notation 3.18 If $\Gamma \in \operatorname{RLG}(G), e \in E(\Gamma)$ and $A$ is an $e$-edge path, we will use the notation $e / A$ to denote the slide move of $e$ over $A$. When we write a composition of slides $e / A \cdot f / B$ we will always assume that $f / B$ is a valid slide move after sliding $e$ over $A$. We have some obvious relations: $e / A \cdot e / A^{\prime}=e / A A^{\prime}$ and $e / \bar{A}$ is the inverse of $e / A$ (here $\bar{A}$ is the reverse of the path $A$ ).

Throughout the rest of the section, $A$ denotes an $e-$ or $\bar{e}$-edge path and $B$ denotes an $f$-or $\bar{f}$-edge path. Likewise for $A^{\prime}, B^{\prime}$, etc. We will use $\alpha$ to denote an $e$ or $\bar{e}$-edge path not containing $f$ or $\bar{f}$, and $\beta$ an $f$ or $\bar{f}$-edge path not containing $e$ or $\bar{e}$.

The following proposition is our current goal.

Proposition 3.19 Suppose $\Gamma \in \operatorname{RLG}^{+}(G)$ and $e, f \in E(\Gamma)(e \neq f, \bar{f})$ where $f$ is nonmobile. Suppose $e / A \cdot f / B$ is valid slide sequence in $\operatorname{RLG}^{+}(G)$. Then:

$$
e / A \cdot f / B=f / B^{\prime} \cdot \bar{f} / B^{\prime \prime} \cdot e / A^{\prime} \cdot \bar{e} / A^{\prime \prime}
$$

for some appropriate edge paths $B^{\prime}, B^{\prime \prime}, A^{\prime}$ and $A^{\prime \prime}$.

We will establish this proposition by a careful analysis of how to commute individual slide moves past one another. We begin by listing several basic relations.

Definition 3.20 In some of the slide relations below, renaming occurs. This does not mean that the edges themselves are renamed. Rather, when the relation is used to substitute some slide moves for others inside a larger sequence of moves, the moves in the larger sequence occurring after the newly substituted moves need to be renamed, so that they still refer to the same edges as before. For example, the instruction "rename $e \mapsto f, f \mapsto \bar{e}$ " means that moves such as $e / \alpha, f / \bar{e}, e / \bar{f}$ occurring later in the sequence should now be written as $f / \alpha, \bar{e} / \bar{f}, f / e$. The reason for this should become clear in the proof of the next lemma.

Lemma 3.21 Suppose that $f$ is nonmobile. Then the following relations are valid:

(a) $e / \alpha \cdot f / \beta=f / \beta \cdot e / \alpha$

(b) $e / \alpha \cdot f / e=f / \bar{\alpha} e \cdot e / \alpha$

(c) $e / \alpha \cdot f / \bar{e}=f / \bar{e} \alpha \cdot e / \alpha$

(d) $e / f \cdot f / \beta=f / \beta \cdot e / \beta f$

(e) $e / f \cdot f / e=f / e$

(f) $e / f \cdot f / \bar{e}=e / f \cdot \bar{e} / f$, then rename $e \mapsto \bar{f}, f \mapsto \bar{e}$

(g) $e / \bar{f} \cdot f / \beta=f / \beta \cdot e / \bar{f} \bar{\beta}$

(h) $e / \bar{f} \cdot f / e=\bar{f} / e$, then rename $e \mapsto f, f \mapsto \bar{e}$

(i) $e / \bar{f} \cdot f / \bar{e}=e / \bar{f}$ 
where $f, \bar{f} \notin \alpha$ and $e, \bar{e} \notin \beta$. Furthermore, after substituting and renaming moves, $f$ still refers to a nonmobile edge.

Proof In the diagrams below, the heavy edge is $f$ and the light edge is $e$. Note that in cases (f) and (h), later references to these edges will be renamed. Since $f$ slides over $e$ or $\bar{e}$ in these cases, $e$ must be nonmobile by Lemma 3.17. So it remains true that $f$ is nonmobile in later moves, after the renaming step.

Now consider the individual cases, recalling that $\alpha$ and $\beta$ do not contain $e, \bar{e}, f$, or $\bar{f}$. Case (a) is obvious. Case (b) is clear after noting that $o(e)=o(\alpha)$ and $t(\alpha)=o(f)$. In case (c) we have $o(\alpha)=o(e)$ and $t(e)=o(f)$ and the relation is clear. In case (d) we have $o(e)=o(f)=o(\beta)$ and the relation is clear.

For (e), shown below, $f$ is a loop at $o(e)$. The labels $\lambda(e), \lambda(f)$ are of the form $c a, a$ since $e$ slides over $f$. Then, since $f$ slides over $e$, we must have $c b \mid a$ (where $b=\lambda(\bar{f})$ ), hence $b \mid a$. Since $f$ is nonmobile, we then have $b=a$. Hence the first slide may simply be omitted.
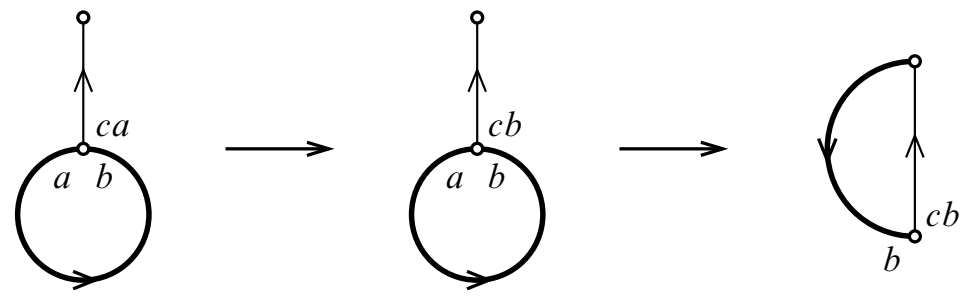

For (f), shown below, $e$ is a loop at $o(f)$ and we have $\lambda(e), \lambda(f)$ of the form $c a, a$ as before. Since $f$ slides over $\bar{e}$, we have $b \mid a$ (where $b=\lambda(\bar{e})$ ) and the new label of $f$ becomes $a c d / b$ (where $d=\lambda(\bar{f})$ ). This integer is divisible by $d$, and so $f$ is now virtually ascending. Since $f$ is nonmobile, we conclude that $a c d / b=d$, so $a c=b$. Since $b \mid a$, we now have $c=1$ and $a=b$. The result of the two moves can now be achieved by sliding $e$ and $\bar{e}$ over $f$. After this move, $\bar{f}$ is in the position previously occupied by $e$, so later references to $e$ should be renamed as $\bar{f}$. Similarly, references to $f$ should be renamed to $\bar{e}$ ( $e$ would work equally well in this case).

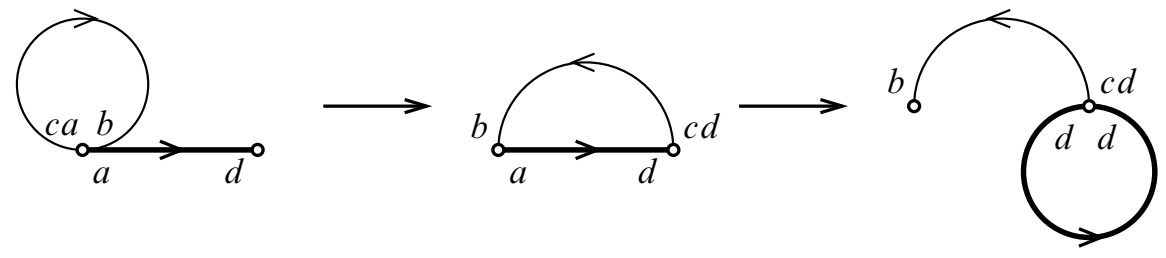


In case (g) we have $o(e)=t(f)$ and $o(f)=o(\beta)$ and the relation is clear. For (h) we have $o(e)=t(f)$, and $\lambda(e), \lambda(\bar{f})$ of the form $b a, a$. After the first slide $\lambda(e)$ becomes $b c$ where $c=\lambda(f)$, and since the second slide occurs we have that $b c \mid c$. Hence $b=1$ and we originally have $\lambda(e)=\lambda(\bar{f})$. Now the same labeled graph results by sliding $\bar{f}$ over $e$. In later moves, $e$ should be renamed as $f$, and $f$ as $\bar{e}$, since $f$ and $\bar{e}$ now occupy the previous positions of $e$ and $f$.

Case (i) is shown below:
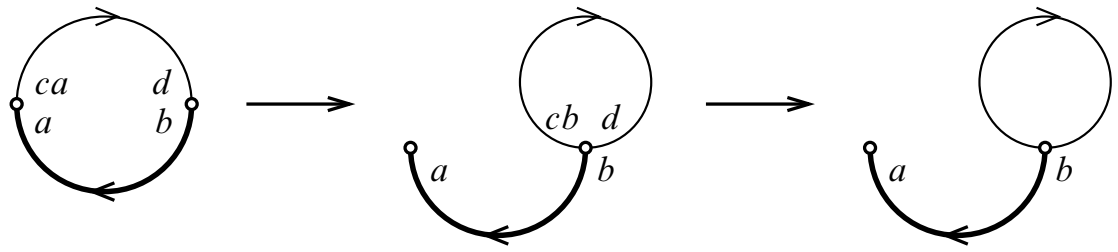

The labels $\lambda(e), \lambda(\bar{f})$ are of the form $c a, a$ and $\lambda(e)$ becomes $c b$ after the first slide (where $b=\lambda(f)$ ). From the second slide we deduce that $d=\lambda(\bar{e})$ divides $b$. Now $e$ is virtually ascending, and it is nonmobile since $f$ can slide over it. So $c b=d$ and the second slide may be omitted.

The next result is a straightforward application of the relations (a)-(d) of Lemma 3.21. It will be used to establish a special case of Proposition 3.19, when either $f, \bar{f} \notin A$ or $e, \bar{e} \notin B$.

Lemma 3.22 Suppose that $f$ is nonmobile. Then the following relations are valid:

(a) $e / \alpha f \alpha^{\prime} \cdot f / \beta=f / \beta \cdot e / \alpha \beta f \alpha^{\prime}$

(b) $e / \alpha \bar{f} \alpha^{\prime} \cdot f / \beta=f / \beta \cdot e / \alpha \bar{f} \bar{\beta} \alpha^{\prime}$

(c) $e / \alpha \cdot f / \beta e \beta^{\prime}=f / \beta \bar{\alpha} e \beta^{\prime} \cdot e / \alpha$

(d) $e / \alpha \cdot f / \beta \bar{e} \beta^{\prime}=f / \beta \bar{e} \alpha \beta^{\prime} \cdot e / \alpha$

Proof For (a) we write (justifying with relations from Lemma 3.21):

$$
\begin{aligned}
e / \alpha f \alpha^{\prime} \cdot f / \beta & =e / \alpha f \cdot f / \beta \cdot e / \alpha^{\prime} & & \text { by (a) } \\
& =e / \alpha \cdot f / \beta \cdot e / \beta f \alpha^{\prime} & & \text { by (d) } \\
& =f / \beta \cdot e / \alpha \beta f \alpha^{\prime} & & \text { by (a). }
\end{aligned}
$$

The other relations are similar. 
The next relations will form the basis of the proof of Proposition 3.19.

Lemma 3.23 Suppose that $f$ is nonmobile. Then the following relations are valid:

(a) $e / \alpha f \alpha^{\prime} \cdot f / \beta e \beta^{\prime}=f / \bar{\alpha} e \beta^{\prime} \cdot e / \alpha \beta$

(b) $e / \alpha f \alpha^{\prime} \cdot f / \beta \bar{e} \beta^{\prime}=f / \beta \cdot \bar{f} / \alpha^{\prime} \cdot e / \alpha \beta f \bar{\alpha}^{\prime} \cdot \bar{e} / f \beta^{\prime}$, rename $e \mapsto \bar{f}, f \mapsto \bar{e}$

(c) $e / \alpha \bar{f} \alpha^{\prime} \cdot f / \beta \bar{e} \beta^{\prime}=f / \alpha^{\prime} \beta^{\prime} \cdot e / \alpha \bar{f} \bar{\beta}^{\prime}$

(d) $e / \alpha \bar{f} \alpha^{\prime} \cdot f / \beta e \beta^{\prime}=f / \alpha^{\prime} \cdot \bar{f} / \bar{\alpha} e \cdot e / \alpha \cdot \bar{e} / \beta^{\prime}$, rename $e \mapsto f, f \mapsto \bar{e}$

Proof The first three of these follow from straightforward computations, similar to Lemma 3.22. Again we justify with relations from Lemma 3.21.

$$
\begin{array}{rlrl}
e / \alpha f \alpha^{\prime} \cdot f / \beta e \beta^{\prime} & =e / \alpha \cdot f / \beta \cdot e / \beta f \cdot f / \bar{\alpha}^{\prime} e \cdot e / \alpha^{\prime} \cdot f / \beta^{\prime} & & \text { by (a), (d), (b) } \\
& =f / \beta \cdot e / \alpha \beta \cdot f / \bar{\alpha}^{\prime} \cdot e / \bar{\alpha}^{\prime} f \cdot f / e \beta^{\prime} \cdot e / \alpha^{\prime} & & \text { by (a), (d) } \\
& =f / \beta \bar{\alpha}^{\prime} \cdot e / \alpha \beta \cdot f / \alpha^{\prime} e \cdot e / \bar{\alpha}^{\prime} \cdot f / \beta^{\prime} \cdot e / \alpha^{\prime} & & \text { by (a), (e), (b) } \\
& =f / \beta \cdot e / \alpha \beta \cdot f / e \beta^{\prime} & & \text { by (a) and } \\
& =f / \bar{\alpha} e \beta^{\prime} \cdot e / \alpha \beta & & \text { cancellation } \\
& \text { by (b), (a). }
\end{array}
$$

This proves (a). For (b) we have, using relations from Lemma 3.21:

$$
\begin{array}{lll}
e / \alpha f \alpha^{\prime} \cdot f / \beta \bar{e} \beta^{\prime} & \\
=e / \alpha f \cdot f / \beta \cdot e / \alpha^{\prime} \cdot f / \bar{e} \beta^{\prime} & \\
=f / \beta \cdot e / \alpha \beta f \cdot f / \bar{e} \alpha^{\prime} \beta^{\prime} \cdot e / \alpha^{\prime} & & \text { by (d), (c), (a) } \\
=f / \beta \cdot e / \alpha \beta f \cdot \bar{e} / f \cdot \bar{e} / \alpha^{\prime} \beta^{\prime} \cdot \bar{f} / \alpha^{\prime}, \text { rename } e \mapsto \bar{f}, f \mapsto \bar{e} & \text { by (f) } \\
=f / \beta \cdot e / \alpha \beta f \cdot \bar{e} / f \cdot \bar{f} / \alpha^{\prime} \cdot \bar{e} / \alpha^{\prime} \beta^{\prime}, \text { rename } e \mapsto \bar{f}, f \mapsto \bar{e} & \text { by (a) } \\
=f / \beta \cdot e / \alpha \beta f \cdot \bar{f} / \alpha^{\prime} \cdot \bar{e} / f \beta^{\prime}, \text { rename } e \mapsto \bar{f}, f \mapsto \bar{e} & & \text { by (g) and } \\
=f / \beta \cdot \bar{f} / \alpha^{\prime} \cdot e / \alpha \beta f \bar{\alpha}^{\prime} \cdot \bar{e} / f \beta^{\prime}, \text { rename } e \mapsto \bar{f}, f \mapsto \bar{e} & & \text { by (g), (a). }
\end{array}
$$

Note that part of the third line has undergone renaming. The renaming instruction is still needed for any subsequent moves. Next consider (c), using relations from Lemma 3.21:

$$
\begin{array}{rlrl}
e / \alpha \bar{f} \alpha^{\prime} \cdot f / \beta \bar{e} \beta^{\prime} & =e / \alpha \bar{f} \cdot f / \beta \cdot e / \alpha^{\prime} \cdot f / \bar{e} \beta^{\prime} & \\
& =f / \beta \cdot e / \alpha \bar{f} \bar{\beta} \alpha^{\prime} \cdot f / \bar{e} \beta^{\prime} & & \text { by (g), (a) } \\
& =f / \beta \cdot e / \alpha \bar{f} \bar{\beta} \cdot f / \bar{e} \alpha^{\prime} \beta^{\prime} \cdot e / \alpha^{\prime} & & \text { by (c), (a) } \\
& =f / \beta \cdot e / \alpha \bar{f} \cdot f / \bar{e} \bar{\beta} \alpha^{\prime} \beta^{\prime} \cdot e / \bar{\beta} \alpha^{\prime} & & \text { by (c), (a) } \\
& =f / \beta \cdot e / \alpha \cdot f / \bar{\beta} \cdot e / \bar{f} \beta \cdot f / \alpha^{\prime} \beta^{\prime} \cdot e / \bar{\beta} \alpha^{\prime} & & \text { by (i), (g) }
\end{array}
$$




$$
\begin{aligned}
& =e / \alpha \bar{f} \cdot f / \alpha^{\prime} \beta^{\prime} \cdot e / \alpha \\
& =f / \alpha^{\prime} \beta^{\prime} \cdot e / \alpha \bar{f} \bar{\beta}^{\prime}
\end{aligned}
$$

by (a) and cancellation by (g), (a) and cancellation.

Finally we prove (d). Notice that as $\alpha \bar{f} \alpha^{\prime}$ is an $e$-edge path $o\left(\alpha^{\prime}\right)=o(f)$ and after sliding $e$ we have that $o(e)=t\left(\alpha^{\prime}\right)$. Also, since $\beta e \beta^{\prime}$ is an $f$-edge path after sliding $e$, $o(\beta)=o(f)=o\left(\alpha^{\prime}\right)$ and $t(\beta)=o(e)=t\left(\alpha^{\prime}\right)$ Therefore, as neither $\alpha^{\prime}$ nor $\beta$ contain $e, \bar{e}, \beta \bar{\alpha}^{\prime}$ is a cycle before sliding $e$. Since after sliding $e$ over $\alpha \bar{f} \alpha^{\prime}$ we can slide $f$ over $\beta e$ we have that $\lambda_{\Gamma}(e) q_{\Gamma}\left(\alpha, \bar{f}, \alpha^{\prime}\right)$ divides $\lambda_{\Gamma}(f) q_{\Gamma}(\beta)$. (Here $\Gamma$ is the labeled graph just before the slide moves under discussion.) In particular, after sliding $\underline{f}$ along $\beta$, we can slide it back along $\bar{\alpha}^{\prime}$. Finally, since $\lambda_{\Gamma}(f)$ divides $\lambda_{\Gamma}(e) q_{\Gamma}(\alpha, \bar{f})$ which divides $\lambda_{\Gamma}(f) q_{\Gamma}\left(\beta \bar{\alpha}^{\prime}\right)$, we have that $q_{\Gamma}\left(\beta \bar{\alpha}^{\prime}\right)$ is an integer. As $f$ is nonmobile, this integer must be 1 (recall that we are assuming that all labels are positive). Hence $f / \beta=f / \alpha^{\prime}$. Now it is easy to verify that (d) is a valid relation, using relations from Lemma 3.21:

$$
\begin{array}{ll}
e / \alpha \bar{f} \alpha^{\prime} \cdot f / \alpha^{\prime} e \beta^{\prime} & \\
=e / \alpha \bar{f} \cdot f / \alpha^{\prime} \cdot e / \alpha^{\prime} \cdot f / e \beta^{\prime} & \\
=f / \alpha^{\prime} \cdot e / \alpha \bar{f} \cdot f / e \beta^{\prime} & \text { by (g), (a) and cancellation } \\
=f / \alpha^{\prime} \cdot e / \alpha \cdot \bar{f} / e \cdot \bar{e} / \beta^{\prime}, \text { rename } e \mapsto f, f \mapsto \bar{e} & \text { by (h) } \\
=f / \alpha^{\prime} \cdot \bar{f} / \bar{\alpha} e \cdot e / \alpha \cdot \bar{e} / \beta^{\prime}, \text { rename } e \mapsto f, f \mapsto \bar{e} & \text { by (b). }
\end{array}
$$

This completes the proof.

We are now in a position to prove Proposition 3.19.

Proof of Proposition 3.19 To simplify the discussion we introduce a shorthand for slide sequences. Slides of the form $e / \alpha$ or $\bar{e} / \alpha$ are denoted by $E$, and those of the form $e / \alpha f \alpha^{\prime}, e / \alpha \bar{f} \alpha^{\prime}, \bar{e} / \alpha f \alpha^{\prime}$ or $\bar{e} / \alpha \bar{f} \alpha^{\prime}$ by $E_{F}$. Likewise define the symbols $F$ and $F_{E}$. Given a slide sequence, let $m$ denote the number of slides of the form $E_{F}$ or $F_{E}$. Let $n$ denote the number of transitions of the form $E_{F} F_{E}$ after omitting the symbols $E, F$. The complexity of the sequence is the pair $(m, n)$, ordered lexicographically.

We are given the sequence $e / A \cdot f / B$, which decomposes into a slide sequence consisting of $E$ 's and $E_{F}$ 's, followed by $F$ 's and $F_{E}$ 's. Our strategy is to apply slide relations to reduce complexity, until $n=0$. If $n=0$ then we have a sequence in which no $E_{F}$ appears before an $F_{E}$. To complete the argument in this case, Lemma 3.21(a) will transform any $E F$ to $F E$; Lemma 3.22((a),(b)) transforms any $E_{F} F$ to $F E_{F}$; and Lemma $3.22((\mathrm{c}),(\mathrm{d}))$ transforms any $E F_{E}$ to $F_{E} E$. Using these relations, the 
sequence can be transformed to one consisting of $F$ 's and $F_{E}$ 's followed by $E$ 's and $E_{F}$ 's. Lastly, since slides of $e$ and $\bar{e}$ (respectively, $f$ and $\bar{f}$ ) commute, the sequence can be put into the desired form $f / B^{\prime} \cdot \bar{f} / B^{\prime \prime} \cdot e / A^{\prime} \cdot \bar{e} / A^{\prime \prime}$.

Next we show how to reduce complexity if $n>0$. We will be applying the relations of Lemma 3.23, some of which involve renaming. When this occurs, the symbols $E_{F}$ and $F_{E}$, and the symbols $E$ and $F$, will be exchanged throughout part of the sequence. Notice that this in itself does not change $m$. Notice also that the relations in Lemma 3.23(a), Lemma 3.23(c) and Lemma 3.23(d) all reduce $m$.

There is one additional rewriting move which has not yet been discussed. The moves $E E_{F}$ may be rewritten either as $E_{F}$ or as $E_{F} E$, depending on whether the edge $e$ appears with the same orientation in the two moves. Similarly, $F_{E} F$ can be rewritten as $F_{E}$ or $F F_{E}$.

The procedure is first to push all $F$ 's to the beginning of the sequence and all $E$ 's to the end, using this last observation and Lemmas Lemma 3.21(a) and Lemma 3.22. This does not change complexity. Then apply relation Lemma 3.23(a), Lemma 3.23(c) or Lemma 3.23(d), if possible, to one of the $E_{F} F_{E}$ pairs, to reduce complexity. If none of these apply, then every $E_{F} F_{E}$ pair matches the left hand side of relation Lemma 3.23(b). Using this relation does not obviously reduce complexity, but we can proceed as follows.

Starting with the rightmost $E_{F} F_{E}$ pair, the slide sequence has the form

$$
\cdots\left(E_{F} F_{E}\right)\left(F_{E}\right)^{*}\left(E_{F}\right)^{*}(E)^{*}
$$

where $^{*}$ denotes zero or more copies of the symbol. Applying Lemma 3.23(b) to this pair, the sequence becomes

$$
\cdots\left(F F E_{F} E_{F}\right)\left(E_{F}\right)^{*}\left(F_{E}\right)^{*}(F)^{*},
$$

with no change to the symbols that are not shown. If the $\left(E_{F}\right)^{*}$ term in the original sequence is empty then $n$ decreases and $m$ stays the same, and complexity has been reduced. Otherwise the new sequence has the same complexity. If this occurs, apply Lemma 3.23 to the newly created rightmost $E_{F} F_{E}$ pair. If case (a), (c) or (d) applies, complexity is reduced as before. If case (b) applies then we are in the situation just discussed, with empty $\left(E_{F}\right)^{*}$ term, and $n$ decreases. Thus, in all cases, complexity has been reduced.

The corollary below follows directly, by repeated application of Proposition 3.19.

Corollary 3.24 Suppose $\Gamma, \Gamma^{\prime}$ are related by a sequence of slides in $\operatorname{RLG}^{+}(G)$ and $f \in E(\Gamma)$ is nonmobile. Then there is a labeled graph $\Gamma_{f} \in \mathrm{S}(\Gamma, f)$ and a sequence of 
slides $\Gamma_{f} \rightarrow \Gamma^{\prime}$ in $\operatorname{RLG}^{+}(G)$ during which the edges $f, \bar{f}$ remain stationary. Moreover, if a geometric edge $e, \bar{e} \in E(\Gamma)$ was stationary in the original slide sequence, then the sequence $\Gamma_{f} \rightarrow \Gamma^{\prime}$ may be chosen to leave $e, \bar{e}$ stationary as well.

\subsection{Finiteness of $\operatorname{RLG}(G)$}

We can now prove Theorem 1.1, along with some applications. Here is a restatement of the theorem.

Theorem 3.25 Let $\Gamma \in \operatorname{RLG}(G)$, where $G \neq B S(1, n)$. Then $|\operatorname{RLG}(G)|=\infty$ if and only if $\Gamma$ has a mobile edge.

Proof Let $G^{+}$be the GBS group represented by the labeled graph $(\Gamma,|\lambda|)$. Changing the signs of a labeling has no effect on divisibility relations, and hence has no effect on slide moves or mobility of edges. Moreover, the absolute value map $\operatorname{RLG}(G) \rightarrow$ $\operatorname{RLG}^{+}\left(G^{+}\right)$is finite-to-one, so $|\operatorname{RLG}(G)|$ is finite if and only if $\left|\operatorname{RLG}^{+}\left(G^{+}\right)\right|$is. Thus, without loss of generality, we may assume that $\Gamma$ is a positive labeled graph, and we may work in $\operatorname{RLG}^{+}(G)$, where Corollary 3.24 is valid.

Suppose $\Gamma$ has a mobile edge $e$. If there is a strict monotone cycle then $G$ is ascending, and since $G \neq B S(1, n)$, it follows that $|\operatorname{RLG}(G)|=\infty$. Otherwise $|\mathrm{S}(\Gamma, e)|=\infty$, which implies that $|\operatorname{RLG}(G)|=\infty$.

Next suppose that $\Gamma$ has no mobile edges. In particular, $G$ is nonascending. By Corollary 2.6, $\operatorname{RLG}^{+}(G)$ is connected by slide moves. Let $e_{1}, e_{2}, \ldots, e_{k}$ be the geometric edges of $\Gamma$. Given any $\Gamma^{\prime} \in \mathrm{RLG}^{+}(G)$, Corollary 3.24 implies that there is a sequence of labeled graphs $\Gamma=\Gamma^{0}, \Gamma^{1}, \ldots, \Gamma^{k}=\Gamma^{\prime}$ such that $\Gamma^{i}$ is in the slide space $\mathrm{S}\left(\Gamma^{i-1}, e_{i}\right)$ for each $i$. Since no $\Gamma^{i}$ has a mobile edge, these slide spaces are all finite, and therefore $\mathrm{RLG}^{+}(G)$ is finite.

Remark 3.26 Since we have an algorithm to determine whether a given labeled graph has a mobile edge (Remark 3.13), the finiteness criterion above can be checked algorithmically.

Example 3.27 Figure 2 shows a labeled graph with modulus a nontrivial integer. For this reason, the finiteness theorem of Forester [7] does not apply. There is only one possible slide move, and the only slide afterwards is its reverse. It follows that there are no mobile edges, by Remark 3.13. Hence this GBS group has only finitely many reduced labeled graphs representing it. 


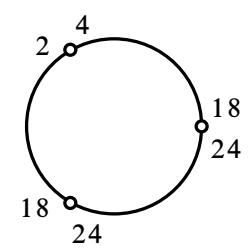

Figure 2: A GBS group with finite labeled graph space and integral modulus

As a consequence of Theorem 1.1, we have the following theorem about the finiteness properties of the group of outer automorphisms of a GBS group. The proof is exactly as in Clay [3, Theorem 75] or Levitt [13, Theorem 1.5]. Recall that a group is of type $\mathrm{F}_{\infty}$ if it is the fundamental group of an aspherical cell complex having finitely many cells in each dimension.

Theorem 3.28 If a GBS group $G$ is represented by a labeled graph that does not have any mobile edges, then $\operatorname{Out}(G)$ is of type $\mathrm{F}_{\infty}$.

Another application concerns the isomorphism problem for GBS groups.

Theorem 3.29 There is an algorithm which, given two labeled graphs, one of which does not have any mobile edges, determines whether the corresponding GBS groups are isomorphic.

Proof Let $\Gamma, \Gamma^{\prime}$ be reduced labeled graphs with corresponding GBS groups $G, G^{\prime}$, where $\Gamma$ has no mobile edges. Remark 3.16 implies that the deformation space of $\Gamma$ is nonascending. Hence, by Corollary 2.6, reduced trees in this deformation space are connected by slide moves (between reduced trees). Since $\operatorname{RLG}(G)$ is finite (Theorem 1.1), it can be enumerated effectively, by performing all possible slide sequences, exactly as in the proof of [7, Corollary 8.3]. Then $G$ and $G^{\prime}$ are isomorphic if and only if the labeled graph $\Gamma^{\prime}$ is found.

\section{Betti number one graphs}

Given a nonelementary GBS group $G$, all labeled graphs $\Gamma \in \operatorname{RLG}(G)$ have the same first Betti number $b(\Gamma)$, since this is simply the rank of the quotient of $G$ by the subgroup generated by the elliptic elements. Alternatively, collapse and expansion moves induce homotopy equivalences of the underlying graph. Thus we also denote this number $b(G)$. In this section we will only consider GBS groups $G$ such that $b(G)=1$. As before, all GBS groups in this section are assumed to be nonelementary. 
Remark 4.1 Suppose $\Gamma \in \operatorname{RLG}(G)$ with $b(\Gamma)=1$. If there is a strict monotone cycle in $\Gamma$, then there is one which is embedded. To see this, note first that there is one which is immersed (by Lemma 3.6). Since $b(\Gamma)=1$, the cycle is a covering of an embedded cycle. Then since the final edge in a monotone cycle appears only once, the cycle itself must be embedded.

Hence, we can determine algorithmically whether a given labeled graph $\Gamma$ with $b(\Gamma)=$ 1 contains a strict monotone cycle.

Proposition 4.2 Suppose $\Gamma \in \operatorname{RLG}(G)$ and $b(\Gamma)=1$. If $\Gamma$ has a strict monotone cycle and $\Gamma^{\prime} \in \operatorname{RLG}(G)$ then $\Gamma^{\prime}$ also has a strict monotone cycle.

Proof By Theorem 2.5 we only need to consider the case when $\Gamma$ and $\Gamma^{\prime}$ are related by a slide, induction or $\mathscr{A}^{ \pm 1}$-move. If $\Gamma$ and $\Gamma^{\prime}$ are related by an induction move, then both contain strict ascending loops and hence both contain strict monotone cycles. Also, if $\Gamma$ and $\Gamma^{\prime}$ are related by an $\mathscr{A}^{ \pm 1}$-move, then one of the labeled graphs contains a strict ascending loop and the other contains a strict virtual ascending loop, hence both contain a strict monotone cycle.

Now assume that $\Gamma$ has an embedded strict monotone cycle $\left(e_{0}, \ldots, e_{n}, e\right)$ and that $\Gamma^{\prime}$ is obtained by sliding an edge $f$ over an edge $f^{\prime}$ in $\Gamma$. Since we can assume that the strict monotone cycle is embedded, $e_{i} \neq e_{j}, \bar{e}_{j}$ for any $i \neq j$. We have several cases to consider depending on the configuration of $f, f^{\prime}$ with respect to the monotone cycle. In all cases, it suffices to find an edge that can be slid into a loop, since it will have the same (integral) modulus as $\left(e_{0}, \ldots, e_{n}, e\right)$ (because $\left.b(\Gamma)=1\right)$.

Clearly if $f, \bar{f} \notin\left(e_{0}, \ldots, e_{n}, e\right)$, then this strict monotone cycle is also a strict monotone cycle in $\Gamma^{\prime}$.

If $f=e_{i}$ and $f^{\prime} \neq \bar{e}$, then $\left(e_{0}, \ldots, e_{i-1}, f^{\prime}, e_{i}, \ldots, e_{n}, e\right)$ is a strict monotone cycle in $\Gamma^{\prime}$. Likewise, if $f=\bar{e}_{i}$ and $f^{\prime} \neq e$ then $\left(e_{0}, \ldots, e_{i}, \bar{f}^{\prime}, e_{i+1}, \ldots, e_{n}, e\right)$ is a strict monotone cycle in $\Gamma^{\prime}$.

Since the strict monotone cycle is embedded, the only possible configurations of $f, f^{\prime}$ where $f \in\left\{e_{i}, \bar{e}_{i}\right\}$ and $f^{\prime} \in\{e, \bar{e}\}$ are when $f=e_{0}$ and $f^{\prime}=\bar{e}$ or $f=\bar{e}_{n}$ and $f^{\prime}=e$. In the first case $\left(e_{1}, \ldots, e_{n}, e_{0}\right)$ is a strict monotone cycle in $\Gamma^{\prime}$. To see this note that $e_{0}$ can slide over $\bar{e}$ and $\bar{e}$ can slide over $e_{0}$, and hence appropriately chosen lifts to the Bass-Serre tree carry the same stabilizer. Then since $\bar{e}$ can slide over the path $\left(e_{0}, \ldots, e_{n}\right)$, we have that $\bar{e}_{0}$ can slide over $\left(e_{1}, \ldots, e_{n}\right)$, after which it becomes a virtual ascending loop. In the second case, with $f=\bar{e}_{n}$ and $f^{\prime}=e$, the path $\left(e_{0}, \ldots, e_{n}\right)$ is a strict monotone cycle in $\Gamma^{\prime}$ for similar reasons. 
The remaining cases of interest are when $f \in\{e, \bar{e}\}$. If $f=e$, then $\left(e_{0}, \ldots, e_{n}, f^{\prime}, e\right)$ is a strict monotone cycle in $\Gamma^{\prime}$, by the following reasoning. For any strict monotone cycle $\left(e_{0}, \ldots, e_{n}, e\right)$ we have that $\lambda(\bar{e}) q\left(e_{0}, \ldots, e_{n}\right)$ is an integer, since this is the label on $\bar{e}$ after sliding over $\left(e_{0}, \ldots, e_{n}\right)$. Also, $\lambda(e)$ divides this integer since the modulus of the cycle is $(\lambda(\bar{e}) / \lambda(e)) q\left(e_{0}, \ldots, e_{n}\right)$. In our situation $\lambda(f)$ divides $\lambda(e)$, and hence also $\lambda(\bar{e}) q\left(e_{0}, \ldots, e_{n}\right)$. So in $\Gamma^{\prime}$ the edge $\bar{e}$ can slide over $\left(e_{0}, \ldots, e_{n}\right)$ and then over $f$.

If $f=\bar{e}$, then $\left(\bar{f}^{\prime}, e_{0}, \ldots, e_{n}, e\right)$ is a strict monotone cycle in $\Gamma^{\prime}$, since $\bar{e}$ can slide back over $\overline{f^{\prime}}$ and then over $\left(e_{0}, \ldots, e_{n}\right)$.

In the case $b(\Gamma)=1$, we now have a converse to the first statement of Lemma 3.6.

Corollary 4.3 If $\Gamma \in \operatorname{RLG}(G)$ satisfies $b(\Gamma)=1$ then $G$ is ascending if and only if $\Gamma$ has an embedded strict monotone cycle.

Proof If $G$ is ascending, then there is a labeled graph $\Gamma^{\prime} \in \operatorname{RLG}(G)$ that contains a strict ascending loop (which is a strict monotone cycle). By Proposition 4.2, $\Gamma$ also contains a strict monotone cycle. The converse is given by Lemma 3.6.

Remark 4.4 Note that the latter condition can be checked effectively by Remark 4.1. Thus, when $b(\Gamma)=1$, we can check algorithmically whether $G$ is ascending. There is no known condition for checking whether a GBS group is ascending in general.

Definition 4.5 A mobile edge that is not an ascending loop or the reverse of an ascending loop is called an $s$-mobile edge ( $s$ stands for "slide"). Note that if $b(\Gamma)=1$ and $e$ is an ascending loop, then sliding $e$ or $\bar{e}$ over another edge always results in a graph that is not reduced. Hence $s$-mobile edges are the only mobile edges that can slide over another edge while staying inside $\operatorname{RLG}(G)$. Given $\Gamma \in \operatorname{RLG}(G)$, let $s(\Gamma)$ be number of geometric $s$-mobile edges. By the following lemma, this number may also be denoted $s(G)$.

Lemma 4.6 If $\Gamma, \Gamma^{\prime} \in \operatorname{RLG}(G)$ and $b(\Gamma)=1$ then $s(\Gamma)=s\left(\Gamma^{\prime}\right)$.

Proof As before, we just need to verify this when $\Gamma$ and $\Gamma^{\prime}$ are related by a slide, induction or $\mathscr{A}^{ \pm 1}$-move. For slide moves, the number of mobile edges is invariant (by Corollary 3.15) and so is the number of ascending loops.

For the other moves, note that at least one of $\Gamma$ or $\Gamma^{\prime}$ must be a single strict ascending loop with trees attached. The $s$-mobile edges are exactly those which can be slid to and around the loop. The result can be verified easily from this description. 


\subsection{The nonmobile subgraph}

Since we are assuming that $b(\Gamma)=1$ for any $\Gamma \in \operatorname{RLG}(G)$, the image $q(G) \subset \mathbb{Q}^{\times}$is generated by a single rational number $q=q(\gamma)$, where $\gamma$ is an (oriented) embedded cycle in $\Gamma$. We may assume that $|q| \geqslant 1$.

Let $\Gamma_{\text {non }} \subset \Gamma$ be the nonmobile subgraph, obtained from $\Gamma$ by discarding the mobile edges and any vertices incident to a strict ascending loop. Note that $\Gamma_{\text {non }}$ may be disconnected, and may have isolated vertices. Let $\Gamma_{1}, \ldots, \Gamma_{k}$ be the simply connected components of $\Gamma_{\text {non }}$. There is at most one component of $\Gamma_{\text {non }}$ not in this list, and this only happens when $G$ is nonascending.

In both of the examples of Figure 3, the nonmobile subgraph consists of the two vertices of valence one; all edges are mobile, and the middle vertex is deleted.

Each $\Gamma_{i}$ carries a subgroup $G_{i}$ of $G$, well defined up to conjugacy. These subgroups and their conjugates will be called nonmobile subgroups.

For each geometric mobile edge $\{e, \bar{e}\}$, one of its orientations will be designated as preferred. If $e \in \gamma$ then $e$ is preferred. Otherwise, if $e, \bar{e} \notin \gamma$, we say $e$ is preferred if $e$ separates $o(e)$ from $\gamma$. Since $b(\Gamma)=1$, exactly one of $e, \bar{e}$ will have this property.

Lemma 4.7 For each simply connected component $\Gamma_{i} \subseteq \Gamma_{\text {non }}$, there is a unique preferred mobile edge $e_{i}$ such that $o\left(e_{i}\right) \in \Gamma_{i}$. Furthermore, every $s$-mobile edge is $e_{i}$ or $\bar{e}_{i}$ for some $i$. Hence $\Gamma_{\text {non }}$ has exactly $s(G)$ simply connected components.

Proof There are two cases depending on whether $\Gamma_{i}$ intersects $\gamma$. If $\Gamma_{i}$ intersects $\gamma$ (which can happen if $\gamma$ contains an $s$-mobile edge), then since $\Gamma_{i}$ is simply connected, there is a (preferred) mobile edge $e_{i} \in \gamma$ such that $o\left(e_{i}\right) \in \Gamma_{i}$. There is at most one other geometric mobile edge in $\gamma$ that is incident to $\Gamma_{i}$. Its preferred orientation must meet $\Gamma_{i}$ in its terminal vertex, since the edges in $\gamma$ are oriented coherently. For any other preferred mobile edge $e^{\prime}$ incident to $\Gamma_{i}$, we have that $\Gamma_{i}$ separates $e^{\prime}$ from $\gamma$, and so $\Gamma_{i}$ contains $t\left(e^{\prime}\right)$, not $o\left(e^{\prime}\right)$.

If $\Gamma_{i}$ does not intersect $\gamma$, then since $b(\Gamma)=1$, there is a unique preferred mobile edge incident to $\Gamma_{i}$, separating $\Gamma_{i}$ from $\gamma$.

For the second statement, let $e$ be a preferred $s$-mobile edge. Then $o(e)$ is not the

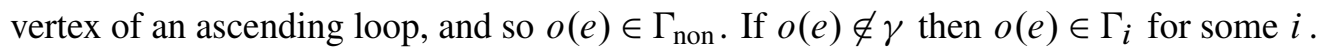
If $o(e) \in \gamma$ then $e \in \gamma$, and hence all components of $\Gamma_{\text {non }}$ are simply connected. Thus $o(e) \in \Gamma_{i}$ for some $i$. In either case, since $o(e) \in \Gamma_{i}$, it follows that $e=e_{i}$ by uniqueness. 
We will be looking carefully at the subgraphs $\Gamma_{i}$ and how they sit inside $\Gamma$. For this we need the following definitions.

Definition 4.8 A pointed labeled graph is a triple $\widehat{\Gamma}=(\Gamma, \hat{v}, \hat{\lambda})$ where $\Gamma$ is a labeled graph, $\hat{v} \in V(\Gamma)$, and $\hat{\lambda}$ is a nonzero integer. It is reduced if $\Gamma$ is reduced and $\widehat{\lambda} \neq \pm 1$. Let $\widehat{\operatorname{RLG}}(G)$ be the set of reduced pointed labeled graphs $(\Gamma, \hat{v}, \hat{\lambda})$ such that $\Gamma \in \operatorname{RLG}(G)$.

We define an equivalence relation on $\widehat{\operatorname{RLG}}(G)$ via the following procedure. Given $(\Gamma, \hat{v}, \hat{\lambda})$, adjoin a new distinguished edge $e$ to $\Gamma$ with $o(e)=\widehat{v}$ and label $\lambda(e)=\hat{\lambda}$ (the label $\lambda(\bar{e})$ is irrelevant). Perform any elementary deformation of this graph in which $e$ is never collapsed. In particular, no edge slides over $e$. Now let $\widehat{v}^{\prime}=o(e)$, $\hat{\lambda}^{\prime}=\lambda(e)$, and delete $e$ to obtain the labeled graph $\Gamma^{\prime}$. If $\Gamma^{\prime}$ is reduced and $\hat{\lambda}^{\prime} \neq \pm 1$, we declare the pointed labeled graphs $(\Gamma, \hat{v}, \hat{\lambda})$ and $\left(\Gamma^{\prime}, \hat{v}^{\prime}, \hat{\lambda}^{\prime}\right)$ to be equivalent.

As always, labeled graphs are considered modulo admissible sign changes, and this applies to pointed labeled graphs as well. In particular, an admissible sign change may be performed on the distinguished edge, and so $(\Gamma, \hat{v}, \hat{\lambda})$ is always equivalent to $(\Gamma, \hat{v},-\hat{\lambda})$. Alternatively, this equivalence can be seen by performing an admissible sign change on every vertex and edge of $\Gamma$.

Given $\widehat{\Gamma} \in \widehat{\operatorname{RLG}}(G)$ let $\widehat{\mathrm{RLG}}(\widehat{\Gamma}) \subset \widehat{\mathrm{RLG}}(G)$ be the equivalence class containing $\widehat{\Gamma}$. It is called the pointed labeled graph space of $\hat{\Gamma}$.

Remark 4.9 It is interesting to compare $\widehat{\mathrm{RLG}}(\widehat{\Gamma})$ with $\operatorname{RLG}(\Gamma)$. For example, let $\widehat{\Gamma}=(\Gamma, \hat{v}, \hat{\lambda})$ where $\Gamma$ is the labeled graph of Figure $2, \hat{v}$ is the upper left vertex, and $\hat{\lambda}=8$. Then $|\widehat{\operatorname{RLG}}(\widehat{\Gamma})|=\infty$ even though $|\operatorname{RLG}(\Gamma)|<\infty$, because the distinguished edge can slide around the cycle in the counterclockwise direction, increasing $\hat{\lambda}$. Indeed, for any labeled graph $\Gamma$ with a nontrivial integral modulus, there will be pointed labeled graphs $\widehat{\Gamma}$ with $|\widehat{R L G}(\widehat{\Gamma})|=\infty$, by similar reasoning. However, if $\Gamma$ has no nontrivial integral moduli, then we have the following result.

Proposition 4.10 Suppose $G$ has no nontrivial integral moduli. Then

(a) $|\widehat{\mathrm{RLG}}(\widehat{\Gamma})|<\infty$ for every $\widehat{\Gamma} \in \widehat{\mathrm{RLG}}(G)$; and

(b) there is an algorithm which, given $\widehat{\Gamma}, \widehat{\Gamma}^{\prime} \in \widehat{\mathrm{RLG}}(G)$, determines whether they are in the same pointed labeled graph space.

Proof Given $\hat{\Gamma}=(\Gamma, \hat{v}, \hat{\lambda})$ let $\Gamma_{0}$ be the reduced labeled graph obtained from $\Gamma$ by adjoining a new edge $e$ and a new vertex $t(e)$, with $o(e)=\hat{v}, \lambda(e)=\hat{\lambda}$, and $\lambda(\bar{e})=2$. Let $G_{0}$ be the new GBS group. This operation does not change the image 
of the modular homomorphism, so $G_{0}$ has no nontrivial integral moduli. Now observe that $\widehat{\mathrm{RLG}}(\widehat{\Gamma})$ embeds into $\operatorname{RLG}\left(G_{0}\right)$, by identifying the distinguished edge with $e$. Conclusion (a) follows because $\operatorname{RLG}\left(G_{0}\right)$ is finite, by [7, Theorem 8.2].

For (b) one considers elementary deformations of $\Gamma_{0}$ in which $e$ is never collapsed. By Corollary 2.6, if $\Gamma_{0}^{\prime}$ is related to $\Gamma_{0}$ by such a deformation, then there is a sequence of slide moves from $\Gamma_{0}$ to $\Gamma_{0}^{\prime}$ in which no edge ever slides over $e$. Now, given $\widehat{\Gamma}$ and $\hat{\Gamma}^{\prime}$, start with $\Gamma_{0}$ and perform all possible sequences of slide moves, never sliding an edge over $e$. All labeled graphs thus obtained yield pointed labeled graphs in $\widehat{\mathrm{RLG}}(\widehat{\Gamma})$ (by recording $o(e)$ and $\lambda(e)$ and deleting $e$ ). Moreover every pointed labeled graph in $\widehat{\mathrm{RLG}}(\widehat{\Gamma})$ will be found, since these slides take place in $\operatorname{RLG}\left(G_{0}\right)$, which is finite.

Definition 4.11 Recall that given $\Gamma \in \operatorname{RLG}(G)$ with $b(\Gamma)=1$, each simply connected component $\Gamma_{i}$ of $\Gamma_{\text {non }}$ has a preferred mobile edge $e_{i}$ associated to it, with $o\left(e_{i}\right) \in \Gamma_{i}$. Define $\widehat{\Gamma}_{i}$ to be the pointed labeled graph $\left(\Gamma_{i}, o\left(e_{i}\right), \lambda\left(e_{i}\right)\right)$. This data will also be denoted $\left(\Gamma_{i}, \widehat{v}_{i}, \hat{\lambda}_{i}\right)$. Note that $\widehat{\Gamma}_{i}$ is reduced, because $\Gamma$ is, and so $\widehat{\Gamma}_{i} \in \widehat{\operatorname{RLG}}\left(G_{i}\right)$ for each $i$.

Theorem 4.12 Suppose $\Gamma, \Gamma^{\prime} \in \operatorname{RLG}(G)$ and $b(\Gamma)=1$. Then

(a) $\Gamma$ and $\Gamma^{\prime}$ define the same nonmobile subgroups of $G$; and

(b) for each nonmobile subgroup $G_{i}$, the corresponding pointed labeled graphs $\widehat{\Gamma}_{i}$ and $\widehat{\Gamma}_{i}^{\prime}$ are equivalent in $\widehat{\operatorname{RLG}}\left(G_{i}\right)$.

Proof We may assume that $\Gamma$ and $\Gamma^{\prime}$ are related by a slide, induction, or $\mathscr{A}$-move.

First consider an induction move. Both labeled graphs have ascending loops, where the move takes place, and note that every edge incident to an ascending loop is mobile. Hence the nonmobile subgraphs and subgroups do not change, nor do the labels $\lambda\left(e_{i}\right)$ (since $o\left(e_{i}\right)$ is not the vertex of the ascending loop).

Next suppose that $\Gamma^{\prime}$ is obtained from $\Gamma$ by an $\mathscr{A}$-move, exactly as pictured in Definition 2.3. The virtually ascending loop in $\Gamma$ with labels $(k, k \ell m)$ is $e_{i}$ for some $i$. Then the vertex of the loop is $\hat{v}_{i}$ and $\hat{\lambda}_{i}=k$. After the $\mathscr{A}$-move, the newly created edge with labels $(k, \ell)$ becomes $e_{i}$, and the subgraph $\Gamma_{i}$ is unchanged. It is still the case that $\hat{\lambda}_{i}=k$, and $\hat{v}_{i}$ has not moved. All other subgraphs $\Gamma_{j}$ are also unchanged. Hence $G_{i}=G_{i}^{\prime}$ and $\widehat{\Gamma}_{i}=\widehat{\Gamma}_{i}^{\prime}$ for all $i$.

Now suppose that $\Gamma^{\prime}$ is obtained from $\Gamma$ by sliding $e$ over $e^{\prime}$. To prove (a) it suffices to show that the simply connected components of $\Gamma_{\text {non }}$ contain the same edges and vertices before and after the slide move. If $e$ is mobile then $\Gamma_{\text {non }}$ does not change at all, 
and (a) holds. So assume that $e$ is nonmobile, which implies that $e^{\prime}$ is also nonmobile, by Lemma 3.17. Now the slide move takes place entirely within $\Gamma_{\text {non }}$, and induces a homotopy equivalence $\Gamma_{\text {non }} \simeq \Gamma_{\text {non }}^{\prime}$ of underlying graphs. Thus the simply connected components are preserved and (a) holds.

Now consider part (b). If $e$ and $e^{\prime}$ are nonmobile then the preferred mobile edges $e_{i}$ do not change, nor do $o\left(e_{i}\right)$ and $\lambda\left(e_{i}\right)$. Thus $\widehat{\Gamma}_{i}$ and $\widehat{\Gamma}_{i}^{\prime}$ are equivalent in $\widehat{\operatorname{RLG}}\left(G_{i}\right)$. If $e$ is mobile then it must be an $s$-mobile edge (cf Definition 4.5) and so $e$ is $e_{i}$ or $\bar{e}_{i}$ for some $i$. If $e=\bar{e}_{i}$ then $o\left(e_{i}\right)$ and $\lambda\left(e_{i}\right)$ do not change, and $\widehat{\Gamma}_{i}=\widehat{\Gamma}_{i}^{\prime}$ for all $i$. Now suppose that $e=e_{i}$. If $e^{\prime}$ is nonmobile and is in $\Gamma_{i}$ for some $i$ then $\hat{\Gamma}_{i}$ and $\hat{\Gamma}_{i}^{\prime}$ are equivalent in $\widehat{\operatorname{RLG}}\left(G_{i}\right)$. If $e^{\prime}$ is nonmobile and not in any $\Gamma_{i}$ then $\widehat{\Gamma}_{i}=\widehat{\Gamma}_{i}^{\prime}$ for each $i$.

Lastly, suppose that $e=e_{i}$ and $e^{\prime}$ is mobile. Note that $o(e)$ is not the vertex of an ascending loop, since $e$ is $s$-mobile and preferred. Hence $e^{\prime}$ is also an $s$-mobile edge. It is not preferred because no two preferred mobile edges have a common initial vertex. Thus $e^{\prime}=\bar{e}_{j}$ for some $j \neq i$. Note that before the slide, $o(e)=o\left(e^{\prime}\right) \in \Gamma_{i}$ and $t\left(e^{\prime}\right) \in \Gamma_{j}$, and after the slide, $o(e)=t\left(e^{\prime}\right) \in \Gamma_{j}$ and $o\left(e^{\prime}\right) \in \Gamma_{i}$. Thus, by the uniqueness property of Lemma 4.7, $e_{i}$ becomes $e_{j}$ and $e_{j}$ becomes $\bar{e}_{i}$. We also have $\widehat{v}_{i}=\widehat{v}_{i}^{\prime}$ and $\widehat{\Gamma}_{i}=\widehat{\Gamma}_{i}^{\prime}$ for all $i$.

The only remaining issue is the labels $\hat{\lambda}_{i}, \hat{\lambda}_{j}$ and $\hat{\lambda}_{i}^{\prime}, \hat{\lambda}_{j}^{\prime}$. We will show that $\lambda(e)=$ $\pm \lambda\left(e^{\prime}\right)$, which implies that $\hat{\lambda}_{i}= \pm \hat{\lambda}_{i}^{\prime}$ and $\hat{\lambda}_{j}= \pm \hat{\lambda}_{j}^{\prime}$, completing the proof. There are two cases.

If $e^{\prime}, \bar{e}^{\prime} \notin \gamma$, then since this is a mobile edge, the geometric edge $\left\{e^{\prime}, \bar{e}^{\prime}\right\}$ can slide to and around $\gamma$ in the positive direction. Since $e^{\prime}$ separates $t\left(e^{\prime}\right)$ from $\gamma$, the endpoint $t\left(e^{\prime}\right)$ can never meet $\gamma$ after sliding $\left\{e^{\prime}, \bar{e}^{\prime}\right\}$. Hence it is $e^{\prime}$, and not $\bar{e}^{\prime}$, which slides to and around $\gamma$. Such a slide sequence includes a slide of $e^{\prime}$ over $e$ (whether $e \in \gamma$ or $e \notin \gamma)$. Also, just before this particular slide, the label $\lambda\left(e^{\prime}\right)$ has not changed, since $o\left(e^{\prime}\right)$ has remained within a subtree of $\Gamma$ until this point. Hence $\lambda(e)$ divides $\lambda\left(e^{\prime}\right)$. On the other hand, since $e$ can slide over $e^{\prime}$, we have that $\lambda\left(e^{\prime}\right)$ divides $\lambda(e)$.

If $\bar{e}^{\prime}=e_{j} \in \gamma$ then write $\gamma$ as $\left(e_{i}, \gamma_{0}, e_{j}\right)$. Note that $\left|\mathrm{S}\left(\Gamma, e^{\prime}\right)\right|<\infty$ since $\Gamma-\left\{e^{\prime}, \bar{e}^{\prime}\right\}$ is a tree. Hence $e^{\prime}$ is part of a strict monotone cycle, which we may take to be embedded, and must then be either $\left(e_{i}, \gamma_{0}, e_{j}\right)$ or $\left(\bar{\gamma}_{0}, \bar{e}_{i}, \bar{e}_{j}\right)$. The second case does not occur since this cycle has modulus $1 / q$, which is not in $\mathbb{Z}-\{ \pm 1\}$. So $\bar{e}_{j}=e^{\prime}$ can slide over $e_{i}=e$, and $\lambda(e)$ divides $\lambda\left(e^{\prime}\right)$. But $e$ can slide over $e^{\prime}$, and so $\lambda\left(e^{\prime}\right)$ divides $\lambda(e)$.

Remark 4.13 It can be shown that conclusion (a) holds even without the assumption that $b(\Gamma)=1$. More specifically, all three types of moves preserve the connected components of the nonmobile subgraph. (Recall from Remark 3.16 that the set of nonmobile edges is preserved by the three moves.) 
Definition 4.14 We may now define an invariant for nonelementary GBS groups $G$ with $b(G)=1$. Choose $\Gamma \in \operatorname{RLG}(G)$ and let $\mathrm{P}(G)$ be the collection of pointed labeled graph spaces $\left\{\widehat{\operatorname{RLG}}\left(\widehat{\Gamma}_{i}\right)\right\}$ indexed by the conjugacy classes of nonmobile subgroups of $G$. By Theorem 4.12, $\mathrm{P}(G)$ is independent of the choice of $\Gamma$.

Moreover $\mathrm{P}(G)$ is computable: given labeled graphs representing $G$ and $G^{\prime}$, one may write down representatives for the collections $\mathrm{P}(G)$ and $\mathrm{P}\left(G^{\prime}\right)$, and determine algorithmically whether $\mathrm{P}(G)=\mathrm{P}\left(G^{\prime}\right)$, by Proposition 4.10.

\subsection{Ascending Betti number one GBS groups}

Let $G$ be an ascending GBS group with $b(G)=1$. Recall that $q(G) \subset \mathbb{Q}^{\times}$is generated by $q=q(\gamma)$ where $\gamma$ is an (oriented) embedded cycle. Since $G$ is ascending, $q \in \mathbb{Z}$ and $|q|>1$. Let $F(q) \subset \mathbb{Q}^{\times}$be the subgroup generated by the integral factors of $q$. We will define an invariant $\xi(G) \in\left(\mathbb{Q}^{\times} /\langle q\rangle\right)^{s} / F(q)$, where $s=s(G)$ and $F(q)$ acts diagonally on the group $\left(\mathbb{Q}^{\times} /\langle q\rangle\right)^{s}$.

Given a labeled graph in $\Gamma \in \operatorname{RLG}(G)$, let $\Gamma_{i}$ and $G_{i}$ be defined as in Section 4.1, and let $e_{1}, \ldots, e_{s}$ be the preferred mobile edges defined by Lemma 4.7. Also choose a mobile edge $e \in \gamma$, called the reference edge. This edge may or may not be among the edges $e_{i}$, depending on whether the strict monotone cycle is an ascending loop. Based on $e$, we will define an element $\xi_{i} \in \mathbb{Q}^{\times} /\langle q\rangle$ for each $G_{i}$, and the resulting $s$-tuple will represent the invariant $\xi(G)$.

First we claim that there are lifts $\widetilde{e}, \tilde{e}_{1}, \ldots, \widetilde{e}_{s}$ in the Bass-Serre tree of $\Gamma$ such that $G_{\widetilde{e}_{i}} \subseteq G_{\widetilde{e}}$ for each $i$. Note that we are free to perform slide moves without affecting this claim. If $e$ is a strict virtually ascending loop, then all mobile edges in $\Gamma$ can be slid to be adjacent to $e$. Then lifts can be chosen so that $t\left(\widetilde{e}_{i}\right)=o(\widetilde{e})$ for each $i$, which implies that $G_{\widetilde{e}_{i}} \subseteq G_{\widetilde{e}}$. Otherwise, if $e$ is not a virtually ascending loop, then it is part of a strict monotone cycle, and it can be made into a virtually ascending loop by slide moves. Now choose lifts as before.

We define $\xi_{i}=\left[G_{\tilde{e}}: G_{\tilde{e}_{i}}\right]$. Note that a different choice of $\tilde{e}_{i}$ defines the same element of $\mathbb{Q}^{\times} /\langle q\rangle$, because the two lifts are related by an element of $G$ with modulus a power of $q$. A different choice of $\tilde{e}$ also makes no difference, by transport of structure. Now define $\xi(\Gamma) \in\left(\mathbb{Q}^{\times} /\langle q\rangle\right)^{s} / F(q)$ to be the element represented by $\left(\xi_{1}, \ldots, \xi_{s}\right)$.

Lemma 4.15 The element $\xi(\Gamma) \in\left(\mathbb{Q}^{\times} /\langle q\rangle\right)^{s} / F(q)$ is independent of the choice of reference edge.

Proof Consider $\xi^{\prime}(\Gamma)$ defined using a reference edge $e^{\prime} \in \gamma$ instead of $e$. We will show that there are lifts $\widetilde{e}, \widetilde{e}^{\prime}$ such that $G_{\widetilde{e}^{\prime}} \subseteq G_{\widetilde{e}}$ and $\left[G_{\widetilde{e}}: G_{\widetilde{e}^{\prime}}\right]$ is a factor of $q$. 
Then the $s$-tuples $\left(\xi_{1}, \ldots, \xi_{s}\right),\left(\xi_{1}^{\prime}, \ldots, \xi_{s}^{\prime}\right) \in\left(\mathbb{Q}^{\times} /\langle q\rangle\right)^{s}$ differ by this factor, and are equivalent in $\left(\mathbb{Q}^{\times} /\langle q\rangle\right)^{s} / F(q)$.

Reversing orientations of $e, e^{\prime}$ if necessary, the cycle $\gamma$ can be written as $\left(\alpha, e, \beta, e^{\prime}\right)$. Both $\left(\alpha, e, \beta, e^{\prime}\right)$ and $\left(\beta, e^{\prime}, \alpha, e\right)$ are strict monotone cycles, because $e$ and $e^{\prime}$ are mobile. Now $\lambda(e)$ divides $q(\alpha) \lambda\left(\bar{e}^{\prime}\right)$, as $(\alpha, e, \beta)$ is an $\bar{e}^{\prime}$-edge path. Similarly $\lambda\left(e^{\prime}\right)$ divides $q(\beta) \lambda(\bar{e})$. Hence the modulus $q$ can be written as the product of two integers:

$$
q=\frac{q(\alpha) \lambda\left(\bar{e}^{\prime}\right)}{\lambda(e)} \frac{q(\beta) \lambda(\bar{e})}{\lambda\left(e^{\prime}\right)} .
$$

Lifting the path $\left(e^{\prime}, \alpha, e\right)$ to $(\widetilde{e}, \tilde{\alpha}, \widetilde{e})$ we obtain $\widetilde{e}$ and $\widetilde{e}^{\prime}$ with $G_{\widetilde{e}^{\prime}} \subseteq G_{\tilde{e}}$. Since $\bar{e}^{\prime}$ can slide over $(\alpha, e)$, we have $\left[G_{\widetilde{e}}: G_{\widetilde{e}^{\prime}}\right]=\left|q(\alpha) \lambda\left(\bar{e}^{\prime}\right) / \lambda(e)\right|$. Hence this index divides $q$.

Next we show that $\xi(\Gamma)$ is an invariant of $G$, and hence may be denoted $\xi(G)$.

Proposition 4.16 For any two graphs $\Gamma, \Gamma^{\prime} \in \operatorname{RLG}(G)$ we have $\xi(\Gamma)=\xi\left(\Gamma^{\prime}\right)$.

Proof By Theorem 2.5, we may assume that $\Gamma^{\prime}$ is obtained from $\Gamma$ by a slide, induction, or $\mathscr{A}^{ \pm 1}$-move. We consider the case of a slide move first.

Since $b(\Gamma)=1$, the slide move does not create or remove strict ascending loops, and so the set of $s$-mobile edges is unchanged. We may also choose a reference edge $e \in \Gamma$ that remains on the embedded circuit in $\Gamma^{\prime}$. Thus, the collection of edges $e, e_{1}, \ldots, e_{s}$ and their lifts, used to define $\xi$, can be chosen to agree for $\Gamma$ and $\Gamma^{\prime}$. The only change to be accounted for in passing from $\Gamma$ to $\Gamma^{\prime}$ is that the correspondence between $s$-mobile edges and conjugacy classes of nonmobile subgroups may change. That is, the indexing of the entries of $\xi(\Gamma)$ may change.

Recall from the proof of Theorem 4.12 that if one $s$-mobile edge slides over another, then their indices and preferred orientations may be exchanged. However, it was shown that whenever this occurs, the labels of the two edges at their common vertex are the same, up to sign. Thus, choosing adjacent lifts $\tilde{e}_{i}$ and $\widetilde{e}_{j}$, we have $G_{\widetilde{e}_{i}}=G_{\widetilde{e}_{j}}$, and therefore $\xi_{i}=\xi_{j}$. It follows that $\xi(\Gamma)=\xi\left(\Gamma^{\prime}\right)$.

If $\Gamma$ and $\Gamma^{\prime}$ differ by an induction move, then there are strict ascending loops $e \in \Gamma$ and $e^{\prime} \in \Gamma^{\prime}$ along which the move occurs. These edges will be the reference edges for $\xi$. The $s$-mobile edges for $\Gamma$ and $\Gamma^{\prime}$ will be the same, with the same indexing, since the move does not affect the nonmobile subgraph. Thus we may choose the same lifts $\tilde{e}_{i}$ for $\Gamma$ and for $\Gamma^{\prime}$. We may also choose the lifts $\tilde{e}$ and $\widetilde{e}^{\prime}$ so that $G_{\tilde{e}} \subseteq G_{\widetilde{e}^{\prime}}$ and $\left[G_{\widetilde{e}^{\prime}}: G_{\widetilde{e}}\right]$ is a factor $m$ of $q$. (Even though $\widetilde{e}$ and $\widetilde{e}^{\prime}$ are in different trees, this can be arranged.) Then $\xi_{i}\left(\Gamma^{\prime}\right)=m \xi_{i}(\Gamma)$ for all $i$, and so $\xi\left(\Gamma^{\prime}\right)=\xi(\Gamma)$. 
Now suppose that $\Gamma^{\prime}$ is obtained from $\Gamma$ by an $\mathscr{A}^{-1}$-move, exactly as pictured in Definition 2.3. In $\Gamma$, the edge with labels $\ell$ and $k$ is an $s$-mobile edge, say $e_{1}$, with initial vertex $v$ on the right. The loop is the reference edge $e$. Choose a lift $\widetilde{e}_{1}$ and let $\widetilde{v}$ be its initial vertex. The $\mathscr{A}^{-1}$-move does not affect $\widetilde{v}$, and the loop $e_{1}^{\prime} \in \Gamma^{\prime}$ has a lift $\tilde{e}_{1}^{\prime}$ with initial vertex $\widetilde{v}$, with the same stabilizer as $\widetilde{e}_{1}$. Note that $e_{1}^{\prime}$ is indeed the $s$-mobile edge in $\Gamma^{\prime}$ corresponding to $G_{1}$. The other nonmobile subgraphs and $s$-mobile edges are unchanged. Thus, the stabilizers of lifts of $s$-mobile edges may be chosen to agree for $\Gamma$ and $\Gamma^{\prime}$. What has changed, however, is the reference edge. The reference edge for $\Gamma^{\prime}$ is $e_{1}^{\prime}$, whose lift $\widetilde{e}_{1}^{\prime}$ has stabilizer $G_{\widetilde{e}_{1}}$. The reference edge for $\Gamma$ is the loop $e$, which has a lift $\tilde{e}$ adjacent to $\widetilde{e}_{1}$, with $G_{\widetilde{e}_{1}} \subseteq G_{\widetilde{e}}$ and $\left[G_{\widetilde{e}}: G_{\widetilde{e}_{1}}\right]=\ell$. Now $\xi_{i}\left(\Gamma^{\prime}\right)=\ell \xi_{i}(\Gamma)$ for all $i$, and $\xi\left(\Gamma^{\prime}\right)=\xi(\Gamma)$, since $\ell$ divides $q$.

Next we define normal forms for the labeled graphs under discussion.

Definition 4.17 Suppose $\Gamma$ is a reduced labeled graph with first Betti number one, in an ascending deformation space. We say that $\Gamma$ is in normal form if it has a strict ascending loop, every mobile edge is adjacent to this loop, and every label (except possibly the label $q$ on the loop) is positive. Note that if $\Gamma$ is in normal form, then the $s$-mobile edges are exactly the edges adjacent to the loop, and $\xi(G)$ is represented by the $s$-tuple $\left(\lambda\left(\bar{e}_{1}\right), \ldots, \lambda\left(\bar{e}_{s}\right)\right)$.

Every $\Gamma$ with $b(\Gamma)=1$ in an ascending deformation space can be put into normal form, as follows. First, there is a strict monotone cycle, which can be made into a strict virtually ascending loop by slide moves. If necessary, this can be made into a strict ascending loop by an $\mathscr{A}$-move. Then all $s$-mobile edges can be slid to be adjacent to the loop. Lastly, since $b(\Gamma)=1$, the labels (other than $q$ ) can be made positive by admissible sign changes.

Example 4.18 Figure 3 shows two reduced labeled graphs in normal form representing groups $G, G^{\prime}$. In both cases the invariant $\xi$ is the equivalence class of the pair $(1,1) \in\left(\mathbb{Q}^{\times} /\langle 2\rangle\right)^{2}$. The invariant $\mathrm{P}(G)$ is represented by a pair of pointed labeled graphs, each consisting of a single vertex, with distinguished labels 2 and 2. On the other hand, $\mathrm{P}\left(G^{\prime}\right)$ is represented by two vertices with distinguished labels 2 and 4 . Thus, we conclude that $G$ and $G^{\prime}$ are not isomorphic. Note that, simple as they are, these two groups are not covered by any of the previously known results on the isomorphism problem (including Theorem 3.29).

Theorem 4.19 Let $G, G^{\prime}$ be ascending Betti number one GBS groups such that $s(G)=s\left(G^{\prime}\right)$ and $q(G)=q\left(G^{\prime}\right)$. Then $G$ and $G^{\prime}$ are isomorphic if and only if their nonmobile subgroups are isomorphic, and under this correspondence between conjugacy classes of nonmobile subgroups, we have $\xi(G)=\xi\left(G^{\prime}\right)$ and $\mathrm{P}(G)=\mathrm{P}\left(G^{\prime}\right)$. 

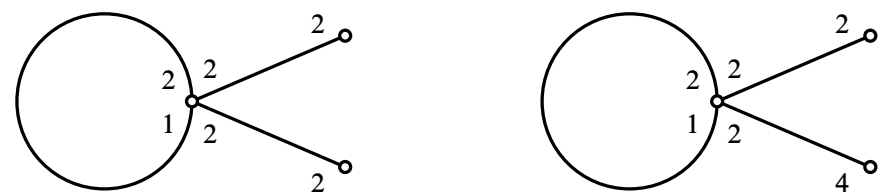

Figure 3: Two nonisomorphic GBS groups

Proof Let $(\Gamma, \lambda)$ and $\left(\Gamma^{\prime}, \lambda^{\prime}\right)$ be reduced labeled graphs in normal form representing $G$ and $G^{\prime}$ respectively. The "only if" direction was proved in Theorem 4.12 and Proposition 4.16. For the other direction we will show that $\Gamma$ and $\Gamma^{\prime}$ are related by a deformation (considered as unmarked labeled graphs), which implies that $G \cong G^{\prime}$.

Let $G_{1}, \ldots, G_{s}$ be the common nonmobile subgroups of $G$ and $G^{\prime}$, and let $\Gamma_{i}$ and $\Gamma_{i}^{\prime}$ be the corresponding components of $\Gamma_{\text {non }}$ and $\Gamma_{\text {non }}^{\prime}$. Then since $\mathrm{P}(G)=\mathrm{P}\left(G^{\prime}\right)$, the pointed labeled graphs $\widehat{\Gamma}_{i}$ and $\widehat{\Gamma}_{i}^{\prime}$ are equivalent in $\widehat{\operatorname{RLG}}\left(G_{i}\right)$. Hence, using the mobile edge $e_{i}$ as the distinguished edge for $\widehat{\Gamma}_{i}$, there is a deformation of $\Gamma$, supported in $\hat{\Gamma}_{i} \cup e_{i}$, making $\hat{\Gamma}_{i}$ isomorphic to $\hat{\Gamma}_{i}^{\prime}$ as pointed labeled graphs. Thus, we may now assume that $\hat{\Gamma}_{i}$ and $\hat{\Gamma}_{i}^{\prime}$ agree for all $i$.

Since the graphs are in normal form, the only possible difference between $\Gamma$ and $\Gamma^{\prime}$ is in the labels $\lambda\left(\bar{e}_{i}\right)$ and $\lambda^{\prime}\left(\bar{e}_{i}\right)$. Since $\xi(G)=\xi\left(G^{\prime}\right)$, the $s$-tuples $\left(\lambda\left(\bar{e}_{1}\right), \ldots, \lambda\left(\bar{e}_{s}\right)\right)$ and $\left(\lambda^{\prime}\left(\bar{e}_{1}\right), \ldots, \lambda^{\prime}\left(\bar{e}_{s}\right)\right)$ are equivalent in $\left(\mathbb{Q}^{\times} /\langle q\rangle\right)^{s} / F(q)$. By performing induction moves, the $s$-tuples can be made equivalent in $\left(\mathbb{Q}^{\times} /\langle q\rangle\right)^{s}$. Now $\lambda\left(\bar{e}_{i}\right)$ and $\lambda^{\prime}\left(\bar{e}_{i}\right)$ differ by a factor of a power of $q$. By slide moves of $\bar{e}_{i}$ over the ascending loop or its reverse, these labels can be made to agree for all $i$.

\subsection{Nonascending Betti number one GBS groups}

Let $G$ be a nonascending GBS group with $b(G)=1$. Suppose also that $G$ is not unimodular, and that the modular group $q(G)$ is generated by an integer $q$. (Otherwise, we are in the situation covered by [7], or alternatively, Theorem 3.29.) For now, we will also assume that $q$ is positive. Let the unique embedded cycle $\gamma \subseteq \Gamma$ be oriented so that $q(\gamma)=q>1$.

An edge has infinite slide space if and only if it can slide to $\gamma$ and around it at least once in the positive direction. If it can slide once all the way around, then it can do so infinitely many times, since its label is multiplied by $q$ each time. No edge can slide infinitely many times around in the negative direction, since no integer is infinitely divisible by $q$. 
Since there are no strict monotone cycles, $\gamma$ does not contain any mobile edges, and hence is contained in a component $\Gamma_{0}$ of $\Gamma_{\text {non }}$. This is the unique component of $\Gamma_{\text {non }}$ that is not simply connected.

Definition 4.20 Let $\Gamma$ be a reduced labeled graph with $b(\Gamma)=1$ in a nonascending, nonunimodular deformation space, with modulus a positive integer. Let $\gamma \subseteq \Gamma$ be the unique embedded cycle, oriented so that $q(\gamma)>1$. We say that $\Gamma$ is in normal form if its labeling is positive and every mobile edge is adjacent to $\gamma$, and cannot slide along $\gamma$ in the negative direction. Clearly, any $\Gamma$ can be put into normal form, by sliding the mobile edges to and along $\bar{\gamma}$ as far as they will go.

Theorem 4.21 Let $G$ be a nonascending, nonunimodular $G B S$ group with $b(G)=1$ and $q(G)$ generated by $q \in \mathbb{Z}_{>0}$. Then $\operatorname{RLG}(G)$ contains only finitely many labeled graphs in normal form, and these can be enumerated effectively from any $\Gamma \in \operatorname{RLG}(G)$.

Proof Suppose $\Gamma^{\prime} \in \operatorname{RLG}(G)$ is in normal form. Let $f_{1}, \ldots, f_{k}$ represent the geometric nonmobile edges of $\Gamma$. By Corollary 3.24 there are sequences of slide moves

$$
\Gamma=\Gamma^{0} \rightarrow \Gamma^{1} \rightarrow \cdots \rightarrow \Gamma^{k} \rightarrow \Gamma^{\prime}
$$

such that the moves $\Gamma^{i-1} \rightarrow \Gamma^{i}$ are slides of $f_{i}, \overline{f_{i}}$ only, and the moves $\Gamma^{k} \rightarrow \Gamma^{\prime}$ are slides of mobile edges only. Thus we have $\Gamma^{i} \in \mathrm{S}\left(\Gamma^{i-1}, f_{i}\right)$ for each $i$, and since each slide space $\mathrm{S}\left(\Gamma^{i-1}, f_{i}\right)$ is finite, there are only finitely many possibilities for the labeled graph $\Gamma^{k}$. These graphs can be found effectively by searching the slide spaces $\mathrm{S}\left(\Gamma^{i-1}, f_{i}\right)$. It now suffices to consider the case when $\Gamma=\Gamma^{k}$, ie when $\Gamma$ and $\Gamma^{\prime}$ are related by slide moves of mobile edges only.

The only ambiguity now in determining $\Gamma^{\prime}$ is in the positioning and labels of the mobile edges, since the nonmobile subgraphs of $\Gamma$ and $\Gamma^{\prime}$ agree. Note that every mobile edge joins $\Gamma_{0}$ to another component $\Gamma_{i}$ (since $\Gamma$ is in normal form). Let $G_{i}$ be the nonmobile subgroup corresponding to $\Gamma_{i}$.

Fix a vertex $v \in \gamma$ and a lift $\tilde{v}$ in the Bass-Serre tree for $\Gamma$. Every mobile edge $\bar{e}_{i}$ may be slid (in the positive direction) along $\gamma$ to $v$, after which the label on $\bar{e}_{i}$ is $n_{i}=\left[G_{\widetilde{v}}: G_{\widetilde{v}} \cap\left(G_{i}\right)^{g}\right]$ for some $g \in G$. Modulo $q$, this index is independent of $g$, so $\left[n_{i}\right] \in \mathbb{Q}^{\times} /\langle q\rangle$ depends only on $\Gamma$ and the choice of $v$.

We claim that in fact, $n_{i}$ itself depends only on the choice of $v$. Namely, no other representative $q^{m} n_{i}$ of $\left[n_{i}\right](m \in \mathbb{Z})$ has the property that an edge $e^{\prime}$ at $v$ with label $q^{m} n_{i}$ can slide around $\gamma$ in the positive direction but not in the negative direction. To see this, slide the edge with smaller label $|m|$ times forward, so the two labels will agree. But then the other edge could have been slid around $\gamma$ in the negative direction. 
Now, once $n_{i}$ is known, the edge $\bar{e}_{i}$ can be slid back to its original position in normal form. This position and the resulting label on $\bar{e}_{i}$ are determined by $n_{i}$. Hence, for any labeled graph in normal form obtained from $\Gamma$ by sliding mobile edges only, the labels and initial endpoints of $\bar{e}_{i}$ are uniquely determined.

It remains to determine the initial vertices $o\left(e_{i}\right)$ and labels $\lambda^{\prime}\left(e_{i}\right)$ in $\Gamma^{\prime}$. The pointed labeled graphs $\hat{\Gamma}_{i}$ and $\hat{\Gamma}_{i}^{\prime}$ have the same underlying labeled graphs, and are equivalent in $\mathrm{P}\left(G_{i}\right)$. Thus, all possible initial vertices $\hat{v}_{i}^{\prime}=o\left(e_{i}\right)$ and labels $\hat{\lambda}_{i}^{\prime}=\lambda^{\prime}\left(e_{i}\right)$ are obtained by sliding the initial endpoint of $e_{i}$ within $\Gamma_{i}$, by Corollary 2.6. Since $\Gamma_{i}$ is simply connected, this slide space is finite and can be searched effectively (cf Proposition 4.10).

We can now prove Theorem 1.2. Recall that this theorem solves the isomorphism problem in the case where one of the labeled graphs has first Betti number at most one.

Proof of Theorem 1.2 Let $\Gamma$ and $\Gamma^{\prime}$ be labeled graphs defining GBS groups $G$ and $G^{\prime}$, where $b(\Gamma) \leqslant 1$. If $q(G)$ is not generated by an integer then the algorithm of [7, Corollary 8.3] determines whether $G \cong G^{\prime}$. Hence we may assume that $b(\Gamma)=1$ and $q(G)$ is generated by $q \in \mathbb{Z}$ with $|q|>1$. We may also assume that $b\left(\Gamma^{\prime}\right)=1$ and $q\left(G^{\prime}\right)=q(G)$, since otherwise $G \not G^{\prime}$. Moveover, we may assume that $q$ is positive, by Lemma 2.7, since the orientation homomorphisms of $\Gamma$ and $\Gamma^{\prime}$ agree.

Now make both graphs reduced by performing collapse moves, and check whether $\Gamma$ and $\Gamma^{\prime}$ are ascending (cf Remark 4.4). If one is ascending and the other is not, the groups are not isomorphic. If both are ascending, then put both into normal form and verify that $s(\Gamma)=s\left(\Gamma^{\prime}\right)$ (if not, then $G \not G^{\prime}$ ). Then identify the subgraphs $\Gamma_{i}, \Gamma_{i}^{\prime}$ and consider permutations $\sigma \in S_{s}$. For each permutation, check whether $G_{i} \cong G_{\sigma(i)}^{\prime}$ for all $i$ (these GBS groups are unimodular, so they can be compared). If so, call $\sigma$ an admissible permutation and then re-index the components of $\Gamma_{\text {non }}^{\prime}$ using $\sigma$, so that $G_{i} \cong G_{i}^{\prime}$ for all $i$. Evaluate and compare the invariants $\xi(G), \xi\left(G^{\prime}\right)$ and $\mathrm{P}(G), \mathrm{P}\left(G^{\prime}\right)$, using Proposition 4.10. By Theorem 4.19, $G$ and $G^{\prime}$ are isomorphic if these invariants agree. If the invariants disagree for every admissible permutation, then $G \nsubseteq G^{\prime}$, again by Theorem 4.19.

If both graphs are nonascending, then put them into normal form. Using Theorem 4.21, enumerate from $\Gamma$ all labeled graphs in $\operatorname{RLG}(G)$ in normal form. Then $G \cong G^{\prime}$ if and only if $\Gamma^{\prime}$ is on this list. 


\section{References}

[1] H Bass, R Kulkarni, Uniform tree lattices, J. Amer. Math. Soc. 3 (1990) 843-902 MR1065928

[2] M Clay, Deformation spaces of G-trees and automorphisms of Baumslag-Solitar groups, to appear in Groups Geom. Dyn.

[3] M Clay, Deformation spaces of $G$-trees, $\mathrm{PhD}$ thesis, University of Utah (2006)

[4] M Clay, M Forester, Whitehead moves for G-trees, to appear in Bull. London Math. Soc.

[5] M Forester, Deformation and rigidity of simplicial group actions on trees, Geom. Topol. 6 (2002) 219-267 MR1914569

[6] M Forester, On uniqueness of JSJ decompositions of finitely generated groups, Comment. Math. Helv. 78 (2003) 740-751 MR2016693

[7] M Forester, Splittings of generalized Baumslag-Solitar groups, Geom. Dedicata 121 (2006) 43-59 MR2276234

[8] N D Gilbert, J Howie, V Metaftsis, E Raptis, Tree actions of automorphism groups, J. Group Theory 3 (2000) 213-223 MR1753479

[9] V Guirardel, G Levitt, Deformation spaces of trees, Groups Geom. Dyn. 1 (2007) 135-181 MR2319455

[10] P H Kropholler, Baumslag-Solitar groups and some other groups of cohomological dimension two, Comment. Math. Helv. 65 (1990) 547-558 MR1078097

[11] P H Kropholler, A note on centrality in 3-manifold groups, Math. Proc. Cambridge Philos. Soc. 107 (1990) 261-266 MR1027778

[12] G Levitt, Characterizing rigid simplicial actions on trees, from: "Geometric methods in group theory”, Contemp. Math. 372, Amer. Math. Soc. (2005) 27-33 MR2139674

[13] G Levitt, On the automorphism group of generalized Baumslag-Solitar groups, Geom. Topol. 11 (2007) 473-515 MR2302496

[14] G Levitt, Personal communication (2008)

[15] M R Pettet, The automorphism group of a graph product of groups, Comm. Algebra 27 (1999) 4691-4708 MR1709254

[16] K Whyte, The large scale geometry of the higher Baumslag-Solitar groups, Geom. Funct. Anal. 11 (2001) 1327-1343 MR1878322

Mathematics Department, University of Oklahoma Norman, OK 73019, USA

mclay@math.ou.edu, forester@math.ou.edu

Received: 10 October 2007 